\title{
Existence of variational solutions to a Cauchy-Dirichlet problem with time-dependent boundary data on metric measure spaces
}

\section{Michael Collins ${ }^{1} \mathbb{D}$}

Received: 10 December 2019 / Accepted: 27 April 2020 / Published online: 23 May 2020

(c) The Author(s) 2020

\begin{abstract}
The objective of this work is an existence proof for variational solutions $u$ to parabolic minimizing problems. Here, the functions being considered are defined on a metric measure space $(\mathcal{X}, d, \mu)$. For such parabolic minimizers that coincide with Cauchy-Dirichlet data $\eta$ on the parabolic boundary of a space-time-cylinder $\Omega \times(0, T)$ with an open subset $\Omega \subset \mathcal{X}$ and $T>0$, we prove existence in the parabolic Newtonian space $L^{p}\left(0, T ; \mathcal{N}^{1, p}(\Omega)\right)$. In this paper we generalize results from Collins and Herán (Nonlinear Anal 176:56-83, 2018) where only time-independent Cauchy-Dirichlet data have been considered. We argue completely on a variational level.
\end{abstract}

Keywords Existence $\cdot$ Variational solutions $\cdot$ Metric measure spaces $\cdot$ Minimizing movements

\section{Introduction}

The aim of this paper is to show existence for parabolic minimizers on metric measure spaces. More precisely, we consider minimizers of integral functionals that are related to scalar functions $u: \Omega \times(0, T) \rightarrow \mathbb{R}$ which satisfy the inequality

$$
\iint u \partial_{t} \varphi \mathrm{d} \mu \mathrm{d} t+\iint f\left(g_{u}\right) \mathrm{d} \mu \mathrm{d} t \leq \iint f\left(g_{u+\varphi}\right) \mathrm{d} \mu \mathrm{d} t
$$

for a continuous integrand $f:[0, \infty] \rightarrow \mathbb{R}$. Here, $\Omega \subset \mathcal{X}$ is a bounded domain, where $(\mathcal{X}, d, \mu)$ is a metric measure space with a metric $d$ and measure $\mu$.

This work was partially supported by the grant DFG-Project HA 7610/1-1 "Existenz- und Regularitätsaussagen für parabolische Quasiminimierer auf metrischen Maßräumen”. Furthermore, the author expresses his gratitude to the Friedrich Naumann Foundation for Freedom that supported him through a postgraduate scholarship.

Michael Collins

collins@math.fau.de

1 Department Mathematik, Friedrich-Alexander-Universität Erlangen-Nürnberg, Cauerstraße 11, 91058 Erlangen, Germany 
In the setting of a metric measure space, the classical calculus known from the Euclidean space $\mathbb{R}^{n}$ is no longer available and instead of the gradient $D u$ we have to introduce the notion of upper gradients. The "minimal upper gradient" will be denoted by $g_{u}$.

The work at hand deals with parabolic minimizers on parabolic domains $\Omega_{T}:=\Omega \times(0, T)$ with $\Omega \subset \mathcal{X}$ bounded and open and $T>0 . \mathcal{X}$ denotes a metric measure space that fulfills a doubling property with respect to the metric $d$ and the measure $\mu$ and supports a suitable Poincaré inequality. We refer to Chapter 2 for exact definitions and the setting of the relevant spaces. Here, we are going to generalize results which have recently been proven in [13], while we follow the methods in [7]. There, the authors have considered an obstacle problem in the Euclidean case.

Since the beginning of the twenty-first century, doubling measure spaces have been studied quite extensively, see for example [10, 18, 25, 26, 28, 30, 39, 53, 54] and especially [3] for an overview and further references. The idea of considering variational problems on metric measure spaces is based on independent proofs of Grigor'yan [23] and Saloff-Coste [51] of the fact that on Riemannian manifolds the doubling property and Poincaré inequality are equivalent to a certain Harnack-type inequality for solutions of the heat equation. But instead of Riemannian manifolds, we are interested in more general spaces.

Existence for parabolic problems on metric measure spaces has already been dealt with in [13] with boundary data independent from time. This paper deals with timedependent boundary data and therefore uses a different approach. In the elliptic case, the Dirichlet problem for $p$-harmonic functions (i.e. $f(\xi)=\xi^{p}$ ) has been considered by Björn and Björn in [3, Chapters 8 and 10] and [4], the latter a joint work with Shanmugalingam. By the results of Cheeger in [10], where it is shown that his definition of Sobolev spaces on metric spaces leads to a reflexive space via the existence of a differential as a measurable section of a finite dimensional cotangent bundle, one can see that direct methods in the calculus of variations can be applied to prove the existence for the $p$-Dirichlet problem, see also [54]. The investigation of parabolic problems on metric measure spaces started not long ago with the work of Kinnunen, Marola, Miranda and Paronetto, see [35], concerning regularity problems. Since then, the most contributions in this field of research have been made to stability theory (see e.g. [20, 21, 43]) and regularity problems (see also [19, 24, 46-48]). When concerning issues of regularity, one tries to establish for instance Hölder continuity of a solution that is assumed to be an element of the parabolic Newtonian space $L^{p}\left(0, T ; \mathcal{N}^{1, p}(\Omega)\right)$. By that function space, we denote those $u:(0, T) \rightarrow \mathcal{N}^{1, p}(\Omega)$, such that the mapping $t \mapsto\|u(t)\|_{\mathcal{N}^{1, p}(\Omega)}^{p}$ is integrable over the interval $(0, T)$.

Our aim is to show existence for a parabolic minimizer of a functional

$$
\mathcal{F}\left(w, \Omega_{T}\right):=\iint_{\Omega_{T}} f\left(g_{w}\right) \mathrm{d} \mu \mathrm{d} t
$$

in such a parabolic Newtonian space, which will be done via the concept of global variational solutions. The integrand is required to be convex and to fulfill a $p$-growth assumption of the form

$$
\nu \xi^{p} \leq f(\xi) \leq L\left(1+\xi^{p}\right)
$$


for all $\xi \in[0, \infty]$ with constants $\nu, L>0,1<p<\infty$. Also, we are going to assume $f$ to be increasing. Again, we refer the reader to Chapter 2 for the exact definitions and in particular to Theorem 2.3 to find the exact statements.

In the paper at hand, we are going to put the focus on how to overcome certain difficulties given by the setting of metric measure spaces. The main difficulty that leads to such obstacles is given by the fact that the upper gradient $g_{u}$ unlike the classical gradient $D u$ does not behave linearly but only in a sub-linear way, such as the modulus $|D u|$ does. In fact, for $\mathcal{X}=\mathbb{R}^{n}$ there holds $g_{u}=|D u|$. Therefore, if we are going to generalize results that have been found for integrands $f: \mathbb{R}^{n} \rightarrow \mathbb{R}$ in the Euclidean case, we have to think about what happens if the integrand only depends on the modulus, i.e. $f(\xi)=g(|\xi|)$ for a convex function $g$. Such integrands have been looked at before in the Euclidean case, see i.e. $[11,12,17,22,44,45]$. Since these papers are dealing with regularity issues, additional assumptions had to be made there, so are the authors in [22] for example looking at integrands $F(|D u|)$ such that $F$ and $\frac{F(t)}{t^{p}}$ are increasing and $\frac{F(t)}{t^{q}}$ is decreasing for $p<q$. In [45] the stationary elliptic case is considered for vector-valued problems, see also [55].

Now for the existence problem, if we require $f: \mathbb{R}^{n} \rightarrow \mathbb{R}$ to be convex, a monotonicity assumption is in order. This assumption (that has also been made in the papers quoted before) comes in a natural way as we can easily see as follows: for $\xi \in \mathbb{R}^{n}$ and $\lambda \in[0,1]$, the convexity of $g$ shows

$$
\begin{aligned}
g(|(1-2 \lambda) \xi|) & =g(|\lambda(-\xi)+(1-\lambda) \xi|) \leq \lambda g(|-\xi|)+(1-\lambda) g(|\xi|) \\
& =\lambda g(|\xi|)+(1-\lambda) g(|\xi|)=g(|\xi|),
\end{aligned}
$$

i.e. by letting $s:=(1-2 \lambda) \in[-1,1]$ we have

$$
g(|s \xi|) \leq g(|\xi|) \text { for } z \in \mathbb{R}^{n} \text { and } s \in[-1,1] .
$$

Now let $\xi_{1}, \xi_{2} \in \mathbb{R}^{n}$ with $0<\left|\xi_{1}\right| \leq\left|\xi_{2}\right|$. Then, for $\xi:=\xi_{2}$ and $s:=\frac{\left|\xi_{1}\right|}{\left|\xi_{2}\right|} \in(0,1]$ we obtain

$$
g\left(\left|\xi_{1}\right|\right)=g\left(\frac{\left|\xi_{1}\right|}{\left|\xi_{2}\right|}\left|\xi_{2}\right|\right) \leq g\left(\left|\xi_{2}\right|\right) .
$$

Hence also in our case, the monotonicity assumption on $f:[0, \infty] \rightarrow \mathbb{R}$ does not impose a loss of generality. These assumptions cover all well known examples, such as the $p$-energy, Orlicz-type integrands and integrands without polynomial structure at all, such as:

$$
\begin{aligned}
\mathcal{F}_{1}[u]: & =\iint_{\Omega_{T}} g_{u}^{p} \mathrm{~d} \mu \mathrm{d} t, \\
\mathcal{F}_{2}[u]: & =\iint_{\Omega_{T}} g_{u}^{p} \log \left(1+g_{u}\right) \mathrm{d} \mu \mathrm{d} t, \\
\mathcal{F}_{3}[u]: & =\iint_{\Omega_{T}} \exp \left(g_{u}^{r}\right) \mathrm{d} \mu \mathrm{d} t .
\end{aligned}
$$

Here, $1<p<\infty$ and $r \geq 1$ are arbitrary integrability exponents.

Our method of proof is aligned to the methods in the work of Bögelein, Duzaar and Scheven [7]. For a fixed step size $h \in(0,1]$ we will inductively construct a sequence $u_{i}$ of minimizers to elliptic variation functionals 


$$
\mathbf{F}_{i}[v]:=\int_{\Omega} f\left(g_{v}\right) \mathrm{d} \mu+\frac{1}{2 h} \int_{\Omega}\left|v-u_{i-1}\right|^{2} \mathrm{~d} \mu
$$

for time-discretized boundary values corresponding to the given boundary data in the Cauchy-Dirichlet problem we are considering. Then, for $t \in((i-1) h, i h]$ we let

$$
u^{(h)}(\cdot, t):=u_{i}
$$

and via certain energy estimates we can show that the limit map $u$ in the sense of weakstar-convergence as $h \searrow 0$ fulfills the variational inequality

$$
\begin{aligned}
\int_{0}^{T} \int_{\Omega} f\left(g_{u}\right) \mathrm{d} \mu \mathrm{d} t \leq & \int_{0}^{T} \int_{\Omega} \partial_{t} v(v-u)+f\left(g_{v}\right) \mathrm{d} \mu \mathrm{d} t \\
& +\frac{1}{2}\left\|v(0)-\eta_{0}\right\|_{L^{2}(\Omega)}^{2}-\frac{1}{2}\|(v-u)(T)\|_{L^{2}(\Omega)}^{2},
\end{aligned}
$$

for any admissible comparison function $v: \Omega_{T} \rightarrow \mathbb{R}$ that coincides with $\eta$ on the lateral boundary $\partial \Omega \times(0, T)$, where $\eta_{0}$ denotes the initial datum. This is called the technique of minimizing movements, see also [1, 15] for further references. To show the energy estimates mentioned before we apply a Jensen-type inequality established in [49]. Finally, to obtain the existence result for more regular data, we introduce a mollification in time.

\section{Setting and statement of results}

\subsection{Notations}

Let $(\mathcal{X}, d, \mu)$ be a separable, connected metric measure space, i.e. $(\mathcal{X}, d)$ is a complete, separable and connected metric space endowed with a Borel measure $\mu$ on $\mathcal{X}$. The measure $\mu$ is assumed to fulfill a doubling property, i.e. there exists a constant $c \geq 1$, such that

$$
0<\mu\left(B_{2 r}(x)\right) \leq c \cdot \mu\left(B_{r}(x)\right)<\infty
$$

for all radii $r>0$ and centres $x \in \mathcal{X}$. Here $B_{r}(x):=\{y \in \mathcal{X}: d(x, y)<r\}$ denotes the open ball with radius $r$ and centre $x$ with respect to the metric $d$. The doubling constant is defined as

$$
c_{d}:=\inf \{c \geq 1:(2.1) \text { holds true }\} .
$$

A complete metric measure space that fulfills the doubling property is proper, meaning that all closed and bounded subsets are compact, see [3, Proposition 3.1].

Following the concept of Cheeger [10], Heinonen and Koskela [30], we call a Borel-function $g: \mathcal{X} \rightarrow[0, \infty]$ an upper gradient for an extended real-valued function $u: \mathcal{X} \rightarrow[-\infty, \infty]$ if for all $x, y \in \mathcal{X}$ and rectifiable curves $\gamma:\left[0, L_{\gamma}\right] \rightarrow \mathcal{X}$ with $\gamma(0)=x, \gamma\left(L_{\gamma}\right)=y$ there holds

$$
|u(x)-u(y)| \leq \int_{\gamma} g \mathrm{~d} s
$$


Moreover, if a non-negative and measurable function $g$ fulfills (2.3) for $p$-almost every curve as before, meaning that the family of curves for which (2.3) fails has $p$-modulus zero, then $g$ is called $p$-weak upper gradient.

For $1 \leq p<\infty$ and a fixed open subset $\Omega \subset \mathcal{X}$ we define the vector space

$$
\tilde{\mathcal{N}}^{1, p}(\Omega):=\left\{u \in L^{p}(\Omega): \exists p \text {-weak upper gradient } g \in L^{p}(\Omega) \text { of } u\right\} .
$$

$L^{p}(\Omega)$ denotes the usual Lebesgue space. The space $\tilde{\mathcal{N}}^{d, p}(\Omega)$ is endowed with the semi-norm

$$
\|u\|_{\tilde{\mathcal{N}}^{1, p}(\Omega)}:=\|u\|_{L^{p}(\Omega)}+\left\|g_{u}\right\|_{L^{p}(\Omega)} .
$$

$g_{u}$ denotes the minimal p-weak upper gradient, i.e. $\left\|g_{u}\right\|_{L^{p}(\Omega)}=\inf \|g\|_{L^{p}(\Omega)}$, where the infimum is taken over all $p$-weak upper gradients of $u$. Introducing the equivalence relation

$$
u \sim v: \Longleftrightarrow\|u-v\|_{\tilde{\mathcal{N}}^{1, p}(\Omega)}=0,
$$

we define the Newtonian space $\mathcal{N}^{1, p}(\Omega)$ as the quotient space

$$
\mathcal{N}^{1, p}(\Omega):=\tilde{\mathcal{N}}^{1, p}(\Omega) / \sim .
$$

Since this definition clearly depends on the metric $d$ and the measure $\mu$, we abuse the notation $\mathcal{N}^{1, p}(\Omega)$ as an abbrevation for $\mathcal{N}^{1, p}(\Omega, d, \mu)$. For more details on metric measure spaces we refer the reader to [3].

As in [3], we define the Sobolev p-capacity of an arbitrary set $E \subset \mathcal{X}$ with respect to the space $\mathcal{N}^{1, p}(\mathcal{X})$ as

$$
\operatorname{Cap}_{p}(E):=\inf \|u\|_{\mathcal{N}^{1, p}(\Omega)}^{p},
$$

where the infimum is taken over all those functions $u \in \mathcal{N}^{1, p}(\mathcal{X})$ such that $\left.u\right|_{E} \geq 1$ holds $\mu$-almost everywhere (in short $\mu$-a.e.). A property is said to hold p-quasi everywhere (in short $p$-q.e.) if the set for which the property fails is of $p$-capacity zero.

We follow the method of [34] and define for an arbitrary set $E \subset \mathcal{X}$ the space $\tilde{\mathcal{N}}_{0}^{1, p}(E)$ as the set of all functions $u: E \rightarrow[-\infty, \infty]$ for which there exists a function $\tilde{u} \in \tilde{\mathcal{N}}^{1, p}(\mathcal{X})$ that fulfills $\tilde{u}=u \mu$-almost everywhere on $E$ and $\tilde{u} \equiv 0 p$-quasi everywhere on $\mathcal{X} \backslash E$. By setting $u \sim v$ if and only if $u=v \mu$-almost everywhere on $E$ we establish an equivalence relation on $\tilde{\mathcal{N}}_{0}^{1, p}(E)$ and define

$$
\mathcal{N}_{0}^{1, p}(E):=\tilde{\mathcal{N}}_{0}^{1, p}(E) / \sim
$$

as the Newtonian space with zero boundary values, equipped with the norm

$$
\|u\|_{\mathcal{N}_{0}^{1, p}(E)}:=\|\tilde{u}\|_{\tilde{\mathcal{N}}^{1, p}(\mathcal{X})} \cdot
$$

\subsection{Parabolic Newtonian spaces}

Since we are looking at problems with time-dependence, we have to give a definition for the parabolic Newtonian space $L^{p}\left(0, T ; \mathcal{N}^{1, p}(\Omega)\right)$ and its local version, respectively. For 
$T>0$, we denote the space-time cylinder over an open subset $\Omega \subset \mathcal{X}$ as $\Omega_{T}:=\Omega \times(0, T)$. The parabolic space

$$
L^{p}\left(0, T ; \mathcal{N}^{1, p}(\Omega)\right)
$$

consists of all functions $u: \Omega_{T} \rightarrow \mathbb{R}$ for which the mapping

$$
\underline{u}:(0, T) \rightarrow \mathcal{N}^{1, p}(\Omega), t \mapsto u(\cdot, t)
$$

is strongly measurable and the function $(0, T) \ni t \mapsto\|u(\cdot, t)\|_{\mathcal{N}^{1, p}(\Omega)}$ is contained in $L^{p}(0, T)$. Here, strongly measurable means measurable in the sense of Bochner, i.e. there exists a sequence of simple functions $\varrho_{k}:(0, T) \rightarrow \mathcal{N}^{1, p}(\Omega)$, such that $\left\|\varrho_{k}(t)-u(\cdot, t)\right\|_{\mathcal{N}^{1, p}(\Omega)} \rightarrow 0$ as $k \rightarrow \infty$ for almost every (a.e.) $t \in(0, T)$. The space $L^{p}\left(0, T ; \mathcal{N}^{1, p}(\Omega)\right)$ is endowed with the norm

$$
\|u\|_{L^{p}\left(0, T ; \mathcal{N}^{1, p}(\Omega)\right)}:=\left(\int_{0}^{T}\|u(\cdot, t)\|_{\mathcal{N}^{1, p}(\Omega)}^{p} \mathrm{~d} t\right)^{\frac{1}{p}} .
$$

Remark 2.1 Since we are dealing with time-dependent problems, the functions $u$ we are concerned with are functions $u \equiv u(x, t)$ depending on the variable $x$ in the metric space $\mathcal{X}$ and on the time variable $t$ in $\mathbb{R}$. Therefore we have to understand the concept of weak upper gradients in the sense of time slices, i.e. for a function $u \in L^{p}\left(0, T ; \mathcal{N}^{1, p}(\Omega)\right)$ the parabolic minimal $p$-weak upper gradient of $u$ is defined as

$$
g_{u}(x, t):=g_{u(\cdot, t)}(x)
$$

for $\left(\mu \otimes \mathcal{L}^{1}\right)$-almost every $(x, t) \in \Omega_{T}$. The resulting map $g_{u}: \Omega_{T} \rightarrow \mathbb{R}$ is $\left(\mu \otimes \mathcal{L}^{1}\right)$-measurable since the spaces $L^{p}\left(0, T ; L^{p}(\Omega)\right)$ and $L^{p}\left(\Omega_{T}\right)$ coincide, see also [13]. This allows us to apply Fubini's theorem to parabolic upper gradients.

Now, following [3, Chapter 2.6], we define the space $L_{\text {loc }}^{p}(\mathcal{X})$ as the set of all functions $u: \mathcal{X} \rightarrow \mathbb{R}$, such that for every $x \in \mathcal{X}$ there exists $r_{x}>0$ with $u \in L^{p}\left(B_{r_{x}}(x)\right)$. Just

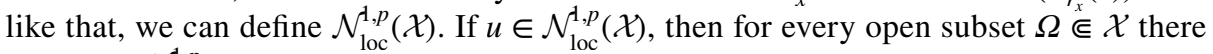
holds $u \in \mathcal{N}^{1, p}(\Omega)$ and moreover, since $\mathcal{X}$ is doubling, the equivalence

$$
u \in \mathcal{N}_{\mathrm{loc}}^{\mathrm{l}, p}(\Omega) \Longleftrightarrow u \in \mathcal{N}^{\mathrm{l}, p}\left(\Omega^{\prime}\right) \text { for all open } \Omega^{\prime} \Subset \Omega
$$

holds for every open subset $\Omega \subset \mathcal{X}$. Now, we can understand the local parabolic space $L_{\text {loc }}^{p}\left(0, T ; \mathcal{N}_{\text {loc }}^{1, p}(\Omega)\right)$ as the set of all functions $u$, such that $u(\cdot, t) \in \mathcal{N}_{\text {loc }}^{1, p}(\Omega)$ for a.e. $t \in(0, T)$ and

$$
\int_{t_{1}}^{t_{2}}\|u(\cdot, t)\|_{\mathcal{N}^{1, p}\left(\Omega^{\prime}\right)}^{p} \mathrm{~d} t<\infty
$$

for all $0<t_{1}<t_{2}<T$ and $\Omega^{\prime} \Subset \Omega$. If clear from the context, we will abbreviate $u(t):=u(\cdot, t)$.

For a Banach space $B$ and $T>0$, the space

$$
C^{0}([0, T] ; B)
$$

consists of all continuous functions $u:[0, T] \rightarrow B$ satisfying 


$$
\|u\|_{C^{0}([0, T] ; B)}:=\max _{0 \leq t \leq T}\|u(t)\|_{B}<\infty .
$$

Naturally, for $\alpha \in(0,1]$, the space

$$
C^{0, \alpha}([0, T] ; B)
$$

consists of those functions $u \in C^{0}([0, T] ; B)$, for which additionally

$$
\sup _{s, t \in[0, T]} \frac{\|u(s)-u(t)\|_{B}}{|s-t|^{\alpha}}<\infty
$$

holds true.

\subsection{Poincaré inequality}

In addition to the doubling property, we demand that the metric measure space $(\mathcal{X}, d, \mu)$ supports a weak $(1, p)$-Poincaré inequality, in the sense that there exist a constant $c_{P}>0$ and a dilatation factor $\tau \geq 1$ such that for all open balls $B_{\rho}\left(x_{0}\right) \subset B_{\tau \rho}\left(x_{0}\right) \subset \mathcal{X}$, for all $L^{p}$ -functions $u$ on $\mathcal{X}$ and all upper gradients $\tilde{g}_{u}$ of $u$ there holds

$$
f_{B_{\rho}\left(x_{0}\right)}\left|u-u_{\rho, x_{0}}\right| \mathrm{d} \mu \leq c_{P} \varrho\left[f_{B_{\tau \rho}\left(x_{0}\right)} \tilde{g}_{u}^{p} \mathrm{~d} \mu\right]^{\frac{1}{p}},
$$

where the symbol

$$
u_{\rho, x_{0}}:=f_{B_{\rho}\left(x_{o}\right)} u \mathrm{~d} \mu:=\frac{1}{\mu\left(B_{\rho}\left(x_{0}\right)\right)} \int_{B_{\rho}\left(x_{o}\right)} u \mathrm{~d} \mu
$$

denotes the mean value integral of the function $u$ on the ball $B_{\rho}\left(x_{0}\right)$ with respect to the measure $\mu$. We will omit the word 'weak' from here on and simply refer to this inequality as 'Poincaré inequality'. Poincaré inequalities on metric measure spaces have been studied quite extensively in the literature, see for example [2, 5, 31, 33, 36, 38, 40, 51, 52].

\subsection{Variational solutions}

In the Euclidean case, i.e. $\mathcal{X}=\mathbb{R}^{n}$, one might consider the Cauchy-Dirichlet problem

$$
\left\{\begin{aligned}
\partial_{t} u-\operatorname{div} D f(D u) & =0 \text { in } \Omega_{T}, \\
u & =\eta \text { on } \partial_{\mathrm{par}} \Omega_{T},
\end{aligned}\right.
$$

where $\partial_{\mathrm{par}} \Omega_{T}:=(\bar{\Omega} \times\{0\}) \cup(\partial \Omega \times(0, T))$ denotes the lateral boundary of $\Omega_{T}$. By an idea of Lichnewsky and Temam [42], one can define the concept of variational solutions to Cauchy-Dirichlet problems like (2.6). Since on a metric measure space we do not have the possibility to explain derivatives such as in (2.6), we cannot consider Cauchy-Dirichlet problems like this. But the concept of variational solutions is described on a purely variational level, so it can be extended to the concept of metric measure spaces. The formulation of the problem takes place in the parabolic function space $\eta+L^{p}\left(0, T ; \mathcal{N}_{0}^{1, p}(\Omega)\right)$, consisting of those $v \in L^{p}\left(0, T ; \mathcal{N}^{1, p}(\Omega)\right)$, such that for almost every time slice $t \in[0, T]$ 
the map $v(t):=v(\cdot, t)$ belongs to the space $\eta(t)+\mathcal{N}_{0}^{1, p}(\Omega)$. See [32, 50] for details on $L^{p}\left(0, T ; \mathcal{N}^{1, p}(\Omega)\right)$. We consider boundary data $\eta: \Omega_{T} \rightarrow \mathbb{R}$ for which there holds

$$
\left\{\begin{array}{c}
\eta \in L^{p}\left(0, T ; \mathcal{N}^{1, p}(\Omega)\right) \text { with } \partial_{t} \eta \in L^{2}\left(\Omega_{T}\right) \\
\text { and } \eta(0)=: \eta_{0} \in L^{2}(\Omega) .
\end{array}\right.
$$

For the convex and continuous integrand $f:[0, \infty] \rightarrow \mathbb{R}$ we assume throughout the whole paper the following $p$-growth and monotonicity conditions

$$
\left\{\begin{array}{c}
\nu \xi^{p} \leq f(\xi) \leq L\left(1+\xi^{p}\right) \text { for all } \xi \in[0, \infty], \\
{[0, \infty] \ni \xi \mapsto f(\xi) \text { is non-decreasing }}
\end{array}\right.
$$

with $v, L>0$. As in [14, Prop. 2.23], we deduce that the convexity of $f$ and the growth condition (2.8) already imply local Lipschitz continuity of $f$ in the sense that

$$
|f(\xi)-f(\zeta)| \leq c(p, L)\left(1+\xi^{p-1}+\zeta^{p-1}\right)|\xi-\zeta|
$$

holds true for all $\xi, \zeta \in[0, \infty]$.

Now, having established a setting, we give the notion of variational solutions of the Cauchy-Dirichlet problem for evolutionary $p$-Laplace type equations on metric measure spaces.

Definition 2.2 (Variational solutions) Assume that the Cauchy-Dirichlet datum $\eta$ fulfills the hypothesis (2.7). Moreover, assume that the integrand $f$ fulfills (2.8). A measurable map $u: \Omega_{T} \rightarrow \mathbb{R}$ in the class

$$
L^{\infty}\left(0, T ; L^{2}(\Omega)\right) \cap\left(\eta+L^{p}\left(0, T ; \mathcal{N}_{0}^{1, p}(\Omega)\right)\right)
$$

is identified as a variational solution to the Cauchy-Dirichlet problem for the gradient flow on the metric measure space $(\mathcal{X}, d, \mu)$ associated to the integrand $f$ if and only if the variational inequality

$$
\begin{aligned}
\int_{0}^{\tau} \int_{\Omega} f\left(g_{u}\right) \mathrm{d} \mu \mathrm{d} t \leq & \int_{0}^{\tau} \int_{\Omega} \partial_{t} v(v-u) \mathrm{d} \mu \mathrm{d} t+\int_{0}^{\tau} \int_{\Omega} f\left(g_{v}\right) \mathrm{d} \mu \mathrm{d} t \\
& -\frac{1}{2}\|(v-u)(\tau)\|_{L^{2}(\Omega)}^{2}+\frac{1}{2}\left\|v(0)-\eta_{0}\right\|_{L^{2}(\Omega)}^{2}
\end{aligned}
$$

holds true for a.e. $\tau \in(0, T)$ and any map $v \in \eta+L^{p}\left(0, T ; \mathcal{N}_{0}^{1, p}(\Omega)\right)$ with $\partial_{t} v \in L^{2}\left(\Omega_{T}\right)$ and $v(0) \in L^{2}(\Omega)$.

We note that by assumption (2.7) the map $\eta$ is an admissible comparison function in the variational inequality (2.10). This allows the deduction of certain energy bounds and shows that variational solutions attend the initial datum $\eta_{0}$ in the $L^{2}$-sense, see below.

Our main result is the following:

Theorem 2.3 Assume that $\eta$ satisfies (2.7). For the convex integrand suppose that the growth condition (2.8) holds true and that $\Omega$ is a bounded domain in $\mathcal{X}$. Then, there exists a variational solution 


$$
u \in L^{\infty}\left(0, T ; L^{2}(\Omega)\right) \cap\left(\eta+L^{p}\left(0, T ; \mathcal{N}_{0}^{1, p}(\Omega)\right)\right)
$$

of the Cauchy-Dirichlet problem in the sense of Definition 2.2. Furthermore, the solution admits the initial datum $\eta_{0}$ in the $L^{2}$-sense.

\section{Preliminaries}

\subsection{Properties of metric measure spaces}

A direct consequence of the doubling property (2.1) is that for every ball $B_{R}(x)$ in $\mathcal{X}$, $y \in B_{R}(x)$ and $0,<r \leq R<\infty$ we have

$$
\frac{\mu\left(B_{r}(y)\right)}{\mu\left(B_{R}(x)\right)} \geq C\left(\frac{r}{R}\right)^{n_{d}},
$$

where $n_{d}:=\log _{2} c_{d}$ and $C:=c_{d}^{-2}$. Moreover, since $\mathcal{X}$ is connected there exists a constant $\tilde{c}>0$ and an exponent $\sigma>0$, such that

$$
\frac{\mu\left(B_{r}(y)\right)}{\mu\left(B_{R}(x)\right)} \leq \tilde{c}\left(\frac{r}{R}\right)^{\sigma} .
$$

The number $n_{d}$ in (3.1) plays the role of a 'dimension from below', related to the measure $\mu$; but since $c_{d}$ in general isn't of the form $2^{m}$ for an integer $m$, we point out that $n_{d}$ cannot expected to be an integer either.

Since $\mathcal{X}$ is a doubling space, it supports the Vitali covering theorem (see [29]) and therefore also the differentiation theorem of Lebesgue, i.e. for every nonnegative locally $\mu$-integrable function on $\mathcal{X}$ we have that

$$
\lim _{r \rightarrow 0} f_{B_{r}(x)} f \mathrm{~d} \mu=f(x)
$$

for $\mu$-almost every $x \in \mathcal{X}$. Now we collect some calculus rules for upper gradients in metric measure spaces. For the proofs, we refer the reader to [3].

Lemma 3.1 (Leibniz rule, Theorem 2.15 in [3]) Let $u, v \in \mathcal{N}_{\mathrm{loc}}^{1, p}(\Omega)$ and let $\tilde{g}_{u}, \tilde{g}_{v} \in L_{\mathrm{loc}}^{p}$ be p-weak upper gradients of $u$ and $v$, respectively. Then the function $\tilde{g}_{u}+\tilde{g}_{v}$ and $|u| \tilde{g}_{v}+|v| \tilde{g}_{u}$ are $p$-weak upper gradients for $u+v$ and $u v$, respectively.

Remark 3.2 Even if we choose the minimal $p$-weak upper gradients $g_{u}$ and $g_{v}$, respectively, the function $|u| g_{v}+|v| g_{u}$ is in general not the minimal $p$-weak upper gradient for $u v$. Counterexamples can be found in the book [3]. This, of course, also applies to the sum $u+v$, which can easily be seen by taking $v=-u$.

Another useful result is the following:

Lemma 3.3 (Theorem 2.18 in [3]) Let $u, v \in \mathcal{N}^{1, p}(\Omega)$ and $\zeta$ be Lipschitz on $\Omega$, such that $0 \leq \zeta \leq 1$. Set $w:=u+\zeta(v-u)=(1-\zeta) u+\zeta v$. Then 


$$
g:=(1-\zeta) g_{u}+\zeta g_{v}+|v-u| g_{\zeta}
$$

is a p-weak upper gradient of $w$.

Remark 3.4 All these rules can be understood also for time-dependent functions $u \in L^{p}\left(0, T ; \mathcal{N}^{1, p}(\Omega)\right)$, as we can define upper gradients for those functions as in (2.4).

Another significant property of minimal $p$-weak upper gradients is that they are local in the sense that two functions coincide on a set, then also their minimal upper gradients coincide on this set. The result is taken from [3, Chapter 2.4].

Lemma 3.5 Let $u, v$ be real-valued functions on $\mathcal{X}$ with minimal upper gradients $g_{u}$ and $g_{v}$, respectively. Then it holds that

$$
g_{u}=g_{v} \quad \mu \text {-almost everywhere on the set }\{x \in \mathcal{X}: u(x)=v(x)\} .
$$

In particular, if $c \in \mathbb{R}$ is a constant, then $g_{u}=0 \mu$-almost everywhere on the set $\{x \in \mathcal{X}: u(x)=c\}$.

\subsection{Poincaré inequalities and Sobolev embedding}

It follows directly from Hölder's inequality that if a metric measure space supports a $(1, p)$-Poincaré inequality, then it supports a $(1, q)$-Poincaré inequality for all $q \geq p$. On the other hand, it was shown in [33] that if a complete metric space is endowed with a doubling measure and supports a $(1, p)$-Poincaré inequality, then it also supports a $(1, p-\varepsilon)$ -Poincaré inequality for some $\varepsilon>0$ (and therefore also a $(1, q)$-Poincaré inequality for all $q \geq p-\varepsilon)$. From [28] we know that if we assume a weak $(1, p)$-Poincaré inequality, then a Sobolev type embedding theorem holds and therefore the space supports a weak $(q, p)$ Poincaré inequality for all $q \leq p^{*}$, with

$$
p^{*}:=\left\{\begin{array}{l}
\frac{n_{d} p}{n_{d}-p}, p<n_{d} \\
+\infty, p \geq n_{d} .
\end{array}\right.
$$

In [37], see also [27, 28, 30], it was shown that in this case for every $u$ in the space $\mathcal{N}^{1, p}\left(B_{2 \tau \rho}\left(x_{0}\right)\right)$ with $B_{2 \tau \rho}\left(x_{0}\right) \subset \mathcal{X}$ the following Sobolev-type inequality holds:

$$
\left[f_{B_{\rho}\left(x_{0}\right)}\left|u-u_{\rho, x_{0}}\right|^{q} \mathrm{~d} \mu\right]^{\frac{1}{q}} \leq c_{*} \varrho\left[f_{B_{2 \tau \rho}\left(x_{0}\right)} g_{u}^{p} \mathrm{~d} \mu\right]^{\frac{1}{p}} \forall 1 \leq q \leq p^{*}
$$

with a constant $c_{*} \equiv c_{*}\left(c_{d}, c_{P}\right)>0$. Poincaré and Sobolev inequalities also hold on more general domains. In detail, the Poincaré inequality holds on bounded measurable subsets $E$ of the metric space $\mathcal{X}$ such that the $p$-capacity of the complement $\mathcal{X} \backslash E$ is positive, i.e. we have

$$
\int_{E}|u|^{p} \mathrm{~d} \mu \leq C_{E} \int_{E} g_{u}^{p} \mathrm{~d} \mu
$$


for every function $u \in \mathcal{N}_{0}^{1, p}(E)$ and for every bounded measurable set $E \subset \mathcal{X}$ with $\mathrm{Cap}_{p}(\mathcal{X} \backslash E)>0$ with a constant $C_{E} \equiv C_{E}\left(c_{P}, c_{d}, p, E\right)>0$.

\subsection{Smoothing procedures in time}

Since variational solutions in the sense of Definition 2.2 are not regular with respect to time, they are in general not admissible as comparison maps in (2.10). To overcome this difficulty, we are going to construct test functions with the correct regularity with respect to time by following an approach introduced by Landes [41]. The idea is to construct the mollification $[v]_{h}^{v_{0}}$, $h>0$, to a given function $v$, such that it (formally) solves the ordinary differential equation

$$
\partial_{t}[v]_{h}^{v_{0}}=-\frac{1}{h}\left([v]_{h}^{v_{0}}-v\right)
$$

with initial condition $[v]_{h}^{v_{0}}(0)=v_{0}$. Precisely, let $B$ be a separable Banach space over $\mathbb{R}$ and $v_{0} \in B$. Now consider $v \in L^{r}(0, T ; B)$ for some $1 \leq r \leq \infty$ and define for $h \in(0, T]$ and $t \in[0, T]$ the mollification in time by

$$
[v]_{h}^{v_{0}}(t):=e^{-\frac{t}{h}} v_{0}+\frac{1}{h} \int_{0}^{t} e^{\frac{s-t}{h}} v(s) \mathrm{d} s .
$$

It is easy to check that $[v]_{h}^{v_{0}}$ satisfies (3.3). For the proof of the following two lemmata, we refer the reader to [6, Appendix B] and [13].

Lemma 3.6 Suppose $B$ is a separable Banach space and $v_{0} \in B$. If $v \in L^{r}(0, T ; B)$ for some $r \geq 1$, then for the mollification $[v]_{h}^{v_{0}}$ defined in (3.4) we have $[v]_{h}^{v_{0}} \in L^{r}(0, T ; B)$ and for any $t_{0} \in(0, T]$ there holds

$$
\left\|[v]_{h}^{v_{0}}\right\|_{L^{r}\left(0, t_{0} ; B\right)} \leq\|v\|_{L^{r}\left(0, t_{0} ; B\right)}+\left[\frac{h}{r}\left(1-e^{-\frac{t_{0} r}{h}}\right)\right]^{\frac{1}{r}}\left\|v_{0}\right\|_{B} .
$$

In the case $r=\infty$ the bracket $[. .]^{\frac{1}{r}}$ in the preceding inequality has to be interpreted as 1. Furthermore, in the case $r<\infty$ we have $[v]_{h}^{v_{0}} \rightarrow v$ in $L^{r}(0, T ; B)$ as $h \searrow 0$. Finally, if $v \in C^{0}([0, T] ; B)$ and $v_{0}=v(0)$, then $[v]_{h}^{v_{0}} \in C^{0}([0, T] ; B), \quad[v]_{h}^{v_{0}}(0)=v_{0}$ and moreover $[v]_{h}^{v_{0}} \rightarrow v$ in $C^{0}([0, T] ; B)$ as $h \searrow 0$.

For maps $v \in L^{r}(0, T ; B)$ with $\partial_{t} v \in L^{r}(0, T ; B)$ we have the following assertion.

Lemma 3.7 Let $B$ be a separable Banach space and $r \geq 1$. Assume that $v \in L^{r}(0, T ; B)$ with $\partial_{t} v \in L^{r}(0, T ; B)$. Then, for the mollification in time defined by

$$
[v]_{h}^{v(0)}(t):=e^{-\frac{t}{h}} v(0)+\frac{1}{h} \int_{0}^{t} e^{\frac{s-t}{h}} v(s) \mathrm{d} s
$$

the time derivative can be computed by

$$
\partial_{t}[v]_{h}^{v_{0}}(t)=\frac{1}{h} \int_{0}^{t} e^{\frac{s-t}{h}} \partial_{s} v(s) \mathrm{d} s
$$

and moreover, we have that 


$$
\left\|\partial_{t}[v]_{h}^{v_{0}}\right\|_{L^{r}(0, T ; B)} \leq\left\|\partial_{t} v\right\|_{L^{r}(0, T ; B)}
$$

holds true.

\subsection{Miscellaneous}

The following results concerning the initial condition $u(0)=\eta_{0}$, energy estimates, continuity in time and uniqueness can be shown analogously to the Euclidean case, see [7]. Note that for our purposes Lemmas 3.6, 3.7 and the following technical lemma that can be proven similarly to [8, Lemma 5.1] by choosing $m=1$ and $u_{0}=\eta_{0}$, see also [9, Lemma 2.4], have taken the place of the lemmata used for the proofs in the Euclidean case.

Lemma 3.8 Assume that $u, \eta \in L^{\infty}\left(0, T ; L^{2}(\Omega)\right)$ are given with $\partial_{t} \eta \in L^{2}\left(\Omega_{T}\right)$. For $h>0$ we define

$$
v_{h}:=[u-\eta]_{h}^{0}+\eta,
$$

where $[u-\eta]_{h}^{0}$ is defined according to (3.4). Then we have

$$
\underset{h \searrow 0}{\limsup } \sup _{\tau \in(0, T)} \int_{0}^{\tau} \int_{\Omega} \partial_{t} v_{h}\left(v_{h}-u\right) \mathrm{d} \mu \mathrm{d} t \leq 0 .
$$

The precise results are:

Lemma 3.9 Assume that $\eta$ satisfies (2.7). Then, any variational solution to the CauchyDirichlet problem in the sense of Definition 2.2 fulfills the initial condition $u(0)=\eta_{0}$ in the sense that

$$
\lim _{h \searrow 0} \frac{1}{h} \int_{0}^{h}\left\|u(t)-\eta_{0}\right\|_{L^{2}(\Omega)}^{2} \mathrm{~d} t=0 .
$$

holds true.

Lemma 3.10 Assume that $u \in L^{\infty}\left(0, T ; L^{2}(\Omega)\right) \cap\left(\eta+L^{p}\left(0, T ; \mathcal{N}_{0}^{1, p}(\Omega)\right)\right)$ is a variational solution of the Cauchy-Dirichlet problem in the sense of Definition 2.2 and that $v \in \eta+L^{p}\left(0, T ; \mathcal{N}_{0}^{1, p}(\Omega)\right)$ is an admissible comparison function, i.e. $\partial_{t} v \in L^{2}\left(\Omega_{T}\right)$ and $v(0) \in L^{2}(\Omega)$. Then the solution $u$ satisfies the energy bound

$$
\begin{aligned}
\sup _{t \in[0, T]} & \|u(t)\|_{L^{2}(\Omega)}^{2}+\int_{0}^{T} \int_{\Omega} f\left(g_{u}\right) \mathrm{d} \mu \mathrm{d} t \\
\leq & 32\left(\int_{0}^{T}\left\|\partial_{t} v(\cdot, t)\right\|_{L^{2}(\Omega)} \mathrm{d} t\right)^{2}+16 \int_{0}^{T} \int_{\Omega} f\left(g_{v}\right) \mathrm{d} \mu \mathrm{d} t \\
& +2 \sup _{t \in[0, T]}\|v(t)\|_{L^{2}(\Omega)}^{2}+8\left\|v(0)-\eta_{0}\right\|_{L^{2}(\Omega)}^{2}
\end{aligned}
$$


Lemma 3.11 Assume that the boundary datum $\eta$ is as in (2.7). Then, every variational solution of the Cauchy-Dirichlet problem in the sense of Definition 2.2 satisfies $u \in C^{0}\left([0, T] ; L^{2}(\Omega)\right)$.

Lemma 3.12 Assume that $\eta$ fulfills (2.7). Then, there is at most one variational solution of the Cauchy-Dirichlet problem with initial and boundary data $\eta$ in the sense of Definition 2.2 .

Note that for the proof of Lemma 3.12, the functions we use as comparison maps in (2.10) are defined as $v_{h}:=[v-\eta]_{h}^{0}+\eta$, similarly as in Lemma (3.8).

Furthermore, it can be shown that a variational solution $u$ to the Cauchy-Dirichlet problem in the sense of Definition 2.2 on the cylinder $\Omega_{T}$ is also a variational solution on sub-cylinders $\Omega_{\tau}$ for any $\tau \in(0, T)$. The proof is also analagous to the Euclidean version, see [7].

\section{Proof of the existence result}

\subsection{Existence for regular data}

In this section we are going to treat the case of sufficiently regular boundary data.

Theorem 4.1 Let $\eta \in L^{p}\left(0, T ; \mathcal{N}^{1, p}(\Omega)\right)$ with

$$
\partial_{t} \eta \in L^{2}\left(\Omega_{T}\right) \cap L^{p}\left(0, T ; \mathcal{N}^{1, p}(\Omega)\right)
$$

and $\eta_{0}:=\eta(0) \in L^{2}(\Omega) \cap \mathcal{N}^{1, p}(\Omega)$ be given. Then there exists a variational solution

$$
u \in \eta+L^{\infty}\left(0, T ; \mathcal{N}_{0}^{1, p}(\Omega)\right) \text { with } \partial_{t} v \in L^{2}\left(\Omega_{T}\right)
$$

to the Cauchy-Dirichlet problem for the gradient flow on the metric measure space $(\mathcal{X}, d, \mu)$ with boundary values $\eta$ in the sense of Definition 2.2. Moreover, there holds

$$
\begin{aligned}
& \frac{1}{2} \int_{0}^{T} \int_{\Omega}\left|\partial_{t} u\right|^{2} \mathrm{~d} \mu \mathrm{d} t+v \sup _{t \in[0, T]} \int_{\Omega} g_{u(\cdot, t)}^{p} \mathrm{~d} \mu \\
& \quad \leq 3 e^{T}\left[L \int_{\Omega}\left(1+g_{\eta_{0}}^{p}\right) \mathrm{d} \mu+\int_{0}^{T} \int_{\Omega}\left[\frac{1}{2}\left|\partial_{t} \eta\right|^{2}+C\left(g_{\partial_{t} \eta}+g_{\partial_{t} \eta}^{p}\right)\right] \mathrm{d} \mu \mathrm{d} t\right]
\end{aligned}
$$

for a constant $C \equiv C(v, L, p)>0$, where $v$ and $L$ are as in the growth condition (2.8).

For the proof of Theorem 4.1 we shall proceed in several steps.

\subsubsection{A sequence of minimizers to elliptic variational functionals}

We fix a step size $h \in(0,1]$ and write $\eta_{i}:=\eta(i h) \in L^{2}(\Omega) \cap \mathcal{N}^{1, p}(\Omega)$ for each $i \in \mathbb{N}_{0}$ with $i h \leq T$ for the time-discretized boundary value as in [7]. Our goal will be to inductively construct a sequence $u_{i} \in L^{2}(\Omega) \cap\left(\eta_{i}+\mathcal{N}_{0}^{1, p}(\Omega)\right)$ of minimizers to certain elliptic variational functionals. The precise construction is as follows. Suppose that $u_{i-1} \in L^{2}(\Omega) \cap\left(\eta_{i-1}+\mathcal{N}_{0}^{1, p}(\Omega)\right)$ for some $i \in \mathbb{N}$ has already been defined. For $i=1, u_{0}=\eta_{0}$ 
shall be the initial boundary datum. Then, we let $u_{i}$ be the minimizer of the variational functional

$$
\mathbf{F}_{i}[v]:=\int_{\Omega} f\left(g_{v}\right) \mathrm{d} \mu+\frac{1}{2 h} \int_{\Omega}\left|v-u_{i-1}\right|^{2} \mathrm{~d} \mu
$$

in the class of functions $v \in L^{2}(\Omega) \cap\left(\eta_{i}+\mathcal{N}_{0}^{1, p}(\Omega)\right)$. Note that this class is non-empty since $v=\eta_{i}$ is admissible. The existence of $u_{i}$ can be deduced by means of standard compactness arguments using the convexity of $f$ combined with the monotonicity condition $(2.8)_{2}$. More detailled, let

$$
\begin{aligned}
\mathcal{K}_{i} & :=\left\{v \in L^{2}(\Omega) \cap\left(\eta_{i}+\mathcal{N}_{0}^{1, p}(\Omega)\right): \mathbf{F}_{i}[v]<\infty\right\}, \\
\|v\| \|_{\mathcal{K}_{i}} & :=\|v\|_{L^{2}(\Omega)}+\|v\|_{\mathcal{N}^{1, p}(\Omega)}
\end{aligned}
$$

and $\left(v_{n}\right)_{n \in \mathbb{N}} \subset \mathcal{K}_{i}$ be a minizing sequence of $\mathbf{F}_{i}$. By making use of the Poincaré inequality (3.2) we can show that there holds

$$
\left\|v_{n}\right\|_{\mathcal{K}_{i}} \leq c\left(\mathbf{F}_{i}\left[\eta_{i}\right]+\mathcal{E}_{i}\right)
$$

for a constant $c \equiv c\left(c_{P}, c_{d}, p, v, h, \Omega\right)>0$ and $\mathcal{E}_{i}:=\left\|u_{i-1}\right\|_{L^{2}(\Omega)}+\left\|\eta_{i}\right\|_{\mathcal{N}^{1, p}(\Omega)}^{p}$. Thus, there is a subsequence of $\left(v_{n}\right)$ that converges weakly to some $v$ in $\mathcal{K}_{i}$ as $n \rightarrow \infty$. By applying Mazur's lemma, we find a sequence $\left(w_{m}\right)_{m \in \mathbb{N}}$ of convex combinations of the $v_{n}$, i.e.

$$
w_{m}=\sum_{n=m}^{l_{m}} a_{n}^{(m)} v_{n}, \sum_{n=m}^{l_{m}} a_{n}^{(m)}=1,
$$

such that $w_{m} \longrightarrow v$ strongly in $\mathcal{K}_{i}$ as $m \rightarrow \infty$, i.e.

$$
\left\{\begin{array}{cc}
w_{m} \longrightarrow v & \text { in } L^{p}(\Omega), \\
g_{w_{m}} \longrightarrow g_{v} & \text { in } L^{p}(\Omega), \\
w_{m} \longrightarrow v & \text { in } L^{2}(\Omega) .
\end{array}\right.
$$

By the convexity of $\mathbf{F}_{i}$, the $w_{m}$ are also a minimizing sequence of $\mathbf{F}_{i}$ in the class $\mathcal{K}_{i}$. By the Lipschitz property (2.9) of $f$ we can deduce lower semicontinuity of $\mathbf{F}_{i}$ with respect to strong convergence in $\mathcal{K}_{i}$, which shows that $v$ is a minimizer of $\mathbf{F}_{i}$ in the class $\mathcal{K}_{i}$. By the strict convexity of the second term in $\mathbf{F}_{i}$, we obtain uniqueness.

\subsubsection{Energy estimates}

We first observe that $u_{i-1}+\eta_{i}-\eta_{i-1}$ is an admissible comparison function in the functional $\mathbf{F}_{i}$ for any $i \in \mathbb{N}$. Therefore, by using the minimality of $u_{i}$ we find that

$$
\begin{aligned}
\mathbf{F}_{i}\left[u_{i}\right] & \leq \mathbf{F}_{i}\left[u_{i-1}+\eta_{i}-\eta_{i-1}\right] \\
& =\frac{1}{2 h} \int_{\Omega}\left|\eta_{i}-\eta_{i-1}\right|^{2} \mathrm{~d} \mu+\int_{\Omega} f\left(g_{u_{i-1}+\eta_{i}-\eta_{i-1}}\right) \mathrm{d} \mu \\
& =\int_{\Omega} f\left(g_{u_{i-1}}\right) \mathrm{d} \mu+\mathbf{I}+\mathbf{I I},
\end{aligned}
$$

where 


$$
\begin{aligned}
\mathbf{I} & :=\frac{1}{2 h} \int_{\Omega}\left|\eta_{i}-\eta_{i-1}\right|^{2} \mathrm{~d} \mu, \\
\mathbf{I I} & :=\int_{\Omega}\left[f\left(g_{u_{i-1}+\eta_{i}-\eta_{i-1}}\right)-f\left(g_{u_{i-1}}\right)\right] \mathrm{d} \mu .
\end{aligned}
$$

For the integral I we have that the estimate

$$
\mathbf{I}=\frac{1}{2 h} \int_{\Omega}\left|\int_{(i-1) h}^{i h} \partial_{t} \eta \mathrm{d} t\right|^{2} \mathrm{~d} \mu \leq \frac{1}{2} \int_{(i-1) h}^{i h} \int_{\Omega}\left|\partial_{t} \eta\right|^{2} \mathrm{~d} \mu \mathrm{d} t
$$

holds true by means of Jensen's inequality. Using in turn the Lipschitz estimate (2.9) for $f$, Young's inequality, the assumption $h \leq 1$ and the growth condition (2.8), we deduce for the integrand of II the following bound from above:

$$
\begin{aligned}
& f\left(g_{u_{i-1}+\eta_{i}-\eta_{i-1}}\right)-f\left(g_{u_{i-1}}\right) \\
& \quad \leq c\left(1+g_{u_{i-1}+\eta_{i}-\eta_{i-1}}^{p-1}+g_{u_{i-1}^{p-1}}^{p-1}\right)\left|g_{u_{i-1}+\eta_{i}-\eta_{i-1}}-g_{u_{i-1}}\right| \\
& \quad \leq c\left(1+g_{u_{i-1}+\eta_{i}-\eta_{i-1}}^{p-1}+g_{u_{i-1}}^{p-1}\right) g_{\eta_{i}-\eta_{i-1}} \\
& \quad \leq c g_{u_{i-1}}^{p-1} g_{\eta_{i}-\eta_{i-1}}+c\left(1+g_{\eta_{i}-\eta_{i-1}}^{p-1}\right) g_{\eta_{i}-\eta_{i-1}} \\
& \quad \leq v h g_{u_{i-1}}^{p}+c\left(1+h^{1-p} g_{\eta_{i}-\eta_{i-1}}^{p-1}\right) g_{\eta_{i}-\eta_{i-1}} \\
& \quad \leq h f\left(g_{u_{i-1}}\right)+c\left(1+h^{1-p} g_{\eta_{i}-\eta_{i-1}}^{p-1}\right) g_{\eta_{i}-\eta_{i-1}}
\end{aligned}
$$

with a constant $c \equiv c(p, L, v)$. Note that there holds

$$
\left(\eta_{i}-\eta_{i-1}\right)(\cdot)=\int_{(i-1) h}^{i h} \partial_{t} \eta(\cdot, t) \mathrm{d} t=h \int_{(i-1) h}^{i h} \partial_{t} \eta(\cdot, t) \mathrm{d} t .
$$

From [49, Theorem 4.1] we therefore obtain the Jensen-type inequality

$$
g_{\eta_{i}-\eta_{i-1}} \leq \int_{(i-1) h}^{i h} g_{\partial_{t} \eta}(\cdot, t) \mathrm{d} t
$$

due to the fact that the mapping $u \mapsto g_{u}$ is convex and $W$-continuous, where $W:=\left\{(u, v) \in L^{p}(\Omega) \times L^{p}(\Omega): u \leq v \mu\right.$-a.e. on $\left.\Omega\right\}$. See [49] for details. By Fubini's theorem, this leads to the estimate

$$
\int_{\Omega} g_{\eta_{i}-\eta_{i-1}} \mathrm{~d} \mu \leq \int_{(i-1) h}^{i h} \int_{\Omega} g_{\partial_{t} \eta} \mathrm{d} \mu \mathrm{d} t
$$

and similarly, by Jensen's inequality there follows

$$
\int_{\Omega} g_{\eta_{i}-\eta_{i-1}}^{p} \mathrm{~d} \mu \leq h^{p-1} \int_{(i-1) h}^{i h} \int_{\Omega} g_{\partial_{t} \eta}^{p} \mathrm{~d} \mu \mathrm{d} t .
$$

Inserting this into II we obtain

$$
\mathbf{I I} \leq h \int_{\Omega} f\left(g_{u_{i-1}}\right) \mathrm{d} \mu+c \int_{(i-1) h}^{i h} \int_{\Omega}\left(g_{\partial_{t} \eta}+g_{\partial_{t} \eta}^{p}\right) \mathrm{d} \mu \mathrm{d} t
$$


for a constant $c \equiv c(p, L, v)$. Together with the estimates for the integral $\mathbf{I}$ this leads to the following preliminary energy estimates

$$
\begin{aligned}
& \int_{\Omega} f\left(g_{u_{i}}\right) \mathrm{d} \mu+\frac{1}{2 h} \int_{\Omega}\left|u_{i}-u_{i-1}\right|^{2} \mathrm{~d} \mu \\
& \quad \leq(1+h) \int_{\Omega} f\left(g_{u_{i-1}}\right) \mathrm{d} \mu \\
& \quad+\int_{(i-1) h}^{i h} \int_{\Omega}\left[\frac{1}{2}\left|\partial_{t} \eta\right|^{2}+c\left(g_{\partial_{t} \eta}+g_{\partial_{t} \eta}^{p}\right)\right] \mathrm{d} \mu \mathrm{d} t .
\end{aligned}
$$

Summing up (4.3) from $i=1, \ldots, l$ for some $l \in \mathbb{N}$ with $l h \leq T$, we conclude that

$$
\begin{aligned}
& \frac{1}{2 h} \sum_{i=1}^{l} \int_{\Omega}\left|u_{i}-u_{i-1}\right|^{2} \mathrm{~d} \mu+\sum_{i=1}^{l} \int_{\Omega} f\left(g_{u_{i}}\right) \mathrm{d} \mu \\
& \quad \leq(1+h) \sum_{i=1}^{l} \int_{\Omega} f\left(g_{u_{i-1}}\right) \mathrm{d} \mu+\int_{0}^{l h} \int_{\Omega}\left[\frac{1}{2}\left|\partial_{t} \eta\right|^{2}+c\left(g_{\partial_{t} \eta}+g_{\partial_{t} \eta}^{p}\right)\right] \mathrm{d} \mu \mathrm{d} t \\
& \quad \leq(1+h) \sum_{i=1}^{l-1} \int_{\Omega} f\left(g_{u_{i}}\right) \mathrm{d} \mu+(1+h) \mathrm{H}(l h) .
\end{aligned}
$$

Here we used the short-hand notation

$$
\mathrm{H}(\tau):=L \int_{\Omega}\left(1+g_{\eta_{0}}^{p}\right) \mathrm{d} \mu+\int_{0}^{\tau} \int_{\Omega}\left[\frac{1}{2}\left|\partial_{t} \eta\right|^{2}+c\left(g_{\partial_{t} \eta}+g_{\partial_{t} \eta}^{p}\right)\right] \mathrm{d} \mu \mathrm{d} t
$$

for $\tau \in(0, T]$. Reabsorbing in the preceding inequality the term

$$
\sum_{i=1}^{l-1} \int_{\Omega} f\left(g_{u_{i}}\right) \mathrm{d} \mu
$$

on the left-hand side, we obtain from the last inequality that

$$
\begin{gathered}
\frac{1}{2 h} \sum_{i=1}^{l} \int_{\Omega}\left|u_{i}-u_{i-1}\right|^{2} \mathrm{~d} \mu+\int_{\Omega} f\left(g_{u_{l}}\right) \mathrm{d} \mu \\
\quad \leq h \sum_{i=1}^{l-1} \int_{\Omega} f\left(g_{u_{i}}\right) \mathrm{d} \mu+(1+h) \mathrm{H}(l h) .
\end{gathered}
$$

Next, we iterate inequality (4.3) from $j=1, \ldots, i$ with $i \in\{1, \ldots, l-1\}$. This leads to

$$
\begin{aligned}
\int_{\Omega} f\left(g_{u_{i}}\right) \mathrm{d} \mu & \leq(1+h)^{i} \int_{\Omega} f\left(g_{\eta_{0}}\right) \mathrm{d} \mu+\sum_{j=1}^{i}(1+h)^{i-j}(\mathrm{H}(j h)-\mathrm{H}((j-1) h)) \\
& \leq(1+h)^{i} \mathrm{H}(i h) \leq(1+h)^{i} \mathrm{H}(l h) .
\end{aligned}
$$

Inserting this into the penultimate inequality yields 


$$
\begin{aligned}
& \frac{1}{2 h} \sum_{i=1}^{l} \int_{\Omega}\left|u_{i}-u_{i-1}\right|^{2} \mathrm{~d} \mu+\int_{\Omega} f\left(g_{u_{l}}\right) \mathrm{d} \mu \\
& \quad \leq h \sum_{i=1}^{l-1}(1+h)^{i} \mathrm{H}(l h)+(1+h) \mathrm{H}(l h)=(1+h)^{l} \mathrm{H}(l h) .
\end{aligned}
$$

Noting that $(1+h)^{l} \leq\left(1+\frac{T}{l}\right)^{l} \leq e^{T}$, the preceding inequality leads to the bound

$$
\frac{1}{2 h} \sum_{i=1}^{l} \int_{\Omega}\left|u_{i}-u_{i-1}\right|^{2} \mathrm{~d} \mu+\int_{\Omega} f\left(g_{u_{l}}\right) \mathrm{d} \mu \leq e^{T} \mathrm{H}(T)
$$

for any $l \in \mathbb{N}$ with $l h \leq T$. From (4.4) we also conclude that

$$
\begin{aligned}
\int_{\Omega}\left|u_{l}\right|^{2} \mathrm{~d} \mu & \leq \int_{\Omega} 2\left[\sum_{i=1}^{l}\left|u_{i}-u_{i-1}\right|\right]^{2} \mathrm{~d} \mu+2 \int_{\Omega}\left|\eta_{0}\right|^{2} \mathrm{~d} \mu \\
& \leq 2 l \sum_{i=1}^{l} \int_{\Omega}\left|u_{i}-u_{i-1}\right|^{2} \mathrm{~d} \mu+2 \int_{\Omega}\left|\eta_{0}\right|^{2} \mathrm{~d} \mu \\
& \leq 4 l h e^{T} \mathrm{H}(T)+2 \|\left.\eta_{0}\right|_{L^{2}(\Omega)} ^{2} \\
& \leq 4 T e^{T} \mathrm{H}(T)+2\left\|\eta_{0}\right\|_{L^{2}(\Omega)}^{2}
\end{aligned}
$$

for any $l \in \mathbb{N}$ with $l h \leq T$.

\subsubsection{The limit map}

From now on, we consider only such values $h \in(0,1]$ for which $l:=\frac{T}{h} \in \mathbb{N}$ holds true. Then, we define a function $u^{(h)}: \Omega \times(-h, T] \rightarrow \mathbb{R}$ by

$$
u^{(h)}(\cdot, t):=u_{i} \text { for } t \in((i-1) h, i h] \text { with } i \in\{0, \ldots, l\} .
$$

From (4.4), (4.5) and the growth condition (2.8) on $f$ we know that

$$
\begin{aligned}
& \sup _{t \in[0, T]}\left\|u^{(h)}(t)\right\|_{L^{2}(\Omega)}^{2}+v \cdot \sup _{t \in[0, T]} \int_{\Omega} g_{u^{(h)}}^{p}(\cdot, t) \mathrm{d} \mu \\
& \quad \leq 2\left\|\eta_{0}\right\|_{L^{2}(\Omega)}^{2}+(1+4 T) e^{T} \mathrm{H}(T)
\end{aligned}
$$

holds true. The energy estimate (4.7) ensures that the sequence $u^{(h)}$ is bounded in $L^{\infty}\left(0, T ; L^{2}(\Omega)\right)$ and $L^{\infty}\left(0, T ; \mathcal{N}^{1, p}\right)$. Moreover, we have that there holds $u^{(h)} \in \eta^{(h)}+L^{\infty}\left(0, T ; \mathcal{N}_{0}^{1, p}(\Omega)\right)$. At this point, some remark on the latter function space is in order. The space $L^{\infty}\left(0, T ; \mathcal{N}^{1, p}(\Omega)\right)$ is isometrically isomorphic to the dual space of $L^{1}\left(0, T ;\left(\mathcal{N}^{1, p}(\Omega)\right)^{*}\right)$, where by $\left(\mathcal{N}^{1, p}(\Omega)\right)^{*}$ we denote the dual space of $\mathcal{N}^{1, p}(\Omega)$. This holds since the space $\mathcal{N}^{1, p}(\Omega)$ is reflexive as a closed subspace of the reflexive Banach space $L^{p}(\Omega)$. See [16, Chapter 4, Section 1] for more details on dual spaces of Bochner spaces. Since $\mathcal{N}^{1, p}(\Omega)$ is also separable for $1 \leq p<\infty$, so is the space $L^{1}\left(0, T ;\left(\mathcal{N}^{1, p}(\Omega)\right)^{*}\right)$. Therefore, there exists a subsequence $\left(h_{k}\right)_{k \in \mathbb{N}}$ with $h_{k} \searrow 0$ as $k \rightarrow \infty$ and a limit function

$$
u \in L^{\infty}\left(0, T ; L^{2}(\Omega)\right) \cap\left(\eta+L^{p}\left(0, T ; \mathcal{N}_{0}^{1, p}(\Omega)\right)\right)
$$


such that in the limit as $k \rightarrow \infty$ there holds

$$
\left\{\begin{array}{l}
u^{\left(h_{k}\right)} \stackrel{*}{*} u \text { weak* in } L^{\infty}\left(0, T ; \mathcal{N}^{1, p}(\Omega)\right), \\
u^{\left(h_{k}\right)} \stackrel{*}{\rightarrow} u \text { weak* in } L^{\infty}\left(0, T ; L^{2}(\Omega)\right) .
\end{array}\right.
$$

Our next goal is to improve the regularity of the limit map $u$. To this end, we define a second function $\tilde{u}^{(h)}: \Omega \times(-h, T] \rightarrow \mathbb{R}$ by linearly interpolating in time the minimizers $u_{i-1}$ and $u_{i}$ on the interval $((i-1) h, i h]$ as in [7], i.e. by letting

$$
\tilde{u}^{(h)}(\cdot, t):=\left(i-\frac{t}{h}\right) u_{i-1}+\left(1-i+\frac{t}{h}\right) u_{i}
$$

for $t \in((i-1) h, i h]$ with $i \in\{1, \ldots, l\}$ and $\tilde{u}^{(h)}(\cdot, t):=\eta_{0}$ for $t \in(-h, 0]$. Note that $\tilde{u}^{(h)}$ and $u^{(h)}$ coincide on $\Omega \times(-h, 0]$ by definition. For $t \in((i-1) h, i h]$ we easily compute the time derivative of $\tilde{u}^{(h)}$ by

$$
\partial_{t} \tilde{u}^{(h)}(\cdot, t)=\frac{1}{h}\left(u_{i}-u_{i-1}\right) \in L^{2}(\Omega) .
$$

Together with (4.4) and the convexity of $f$ this implies

$$
\frac{1}{2} \int_{0}^{T} \int_{\Omega}\left|\partial_{t} \tilde{u}^{(h)}\right|^{2} \mathrm{~d} \mu \mathrm{d} t+v \cdot \sup _{t \in[0, T]} \int_{\Omega} g_{\tilde{u}^{(h)}}^{p}(\cdot, t) \mathrm{d} \mu \leq 3 e^{T} \mathrm{H}(T) .
$$

Therefore, also the family of $\tilde{u}^{(h)}$ is bounded in $L^{\infty}\left(0, T ; \mathcal{N}^{1, p}(\Omega)\right)$ and the family of $\partial_{t} \tilde{u}^{(h)}$ is bounded in $L^{2}\left(\Omega_{T}\right)$. Hence, there exists a subsequence $\left(h_{k}\right)_{k \in \mathbb{N}}$ with $h_{k} \searrow 0$ as $k \rightarrow \infty$ and a limit function $\tilde{u} \in L^{\infty}\left(0, T ; \mathcal{N}^{1, p}(\Omega)\right)$ with $\partial_{t} \tilde{u} \in L^{2}\left(\Omega_{T}\right)$ such that

$$
\left\{\begin{array}{cl}
\tilde{u}^{\left(h_{k}\right)} \rightarrow \tilde{u} & \text { strongly in } L^{\min \{2, p\}}\left(\Omega_{T}\right), \\
\tilde{u}^{\left(h_{k}\right)} \stackrel{*}{\sim} \tilde{u} & \text { weak } \operatorname{in} L^{\infty}\left(0, T ; \mathcal{N}^{1, p}(\Omega)\right), \\
\partial_{t} \tilde{u}^{\left(h_{k}\right)} \rightarrow \partial_{t} \tilde{u} & \text { weakly in } L^{2}\left(\Omega_{T}\right)
\end{array}\right.
$$

holds in the limit $k \rightarrow \infty$. The strong convergence in $L^{\min \{2, p\}}\left(\Omega_{T}\right)$ is secured by a metric version of the Rellich-Kondrachov theorem, see [43, Theorem 4.1]. This theorem can be applied since we assumed the doubling condition (2.1) and the weak (1,p)-Poincare inequality (2.5). Since we have

$$
\left|\left(\tilde{u}^{(h)}-u^{(h)}\right)(t)\right| \leq\left|u_{i}-u_{i-1}\right| \text { for } t \in((i-1) h, i h],
$$

we conclude from (4.4) that

$$
\int_{0}^{T} \int_{\Omega}\left|\tilde{u}^{(h)}-u^{(h)}\right|^{2} \mathrm{~d} \mu \mathrm{d} t \leq h \sum_{i=1}^{\frac{T}{h}} \int_{\Omega}\left|u_{i}-u_{i-1}\right|^{2} \mathrm{~d} \mu \leq 2 h^{2} e^{T} \mathrm{H}(T),
$$

which by means of Hölder's inequality implies

$$
\int_{0}^{T} \int_{\Omega}\left|\tilde{u}^{(h)}-u^{(h)}\right|^{\min \{2, p\}} \mathrm{d} \mu \mathrm{d} t \leq\left(2 h^{2} e^{T} \mathrm{H}(T)\right)^{\frac{\min \{2, p\}}{2}}(T \mu(\Omega))^{1-\frac{\min \{2, p\}}{2}} .
$$

Joining this with (4.10) $)_{1}$ implies that also $u^{\left(h_{k}\right)} \rightarrow \tilde{u}$ strongly in $L^{\min \{2, p\}}\left(\Omega_{T}\right)$ as $k \rightarrow \infty$ and hence $\tilde{u}=u$. By weak lower semicontinuity and (4.9) we infer that 


$$
\frac{1}{2} \int_{0}^{T} \int_{\Omega}\left|\partial_{t} u\right|^{2} \mathrm{~d} \mu \mathrm{d} t+v \cdot \sup _{t \in[0, T]} \int_{\Omega} g_{u}^{p}(\cdot, t) \mathrm{d} \mu \leq 3 e^{T} \mathrm{H}(T) .
$$

By definition of $\mathrm{H}$, this yields the claimed energy estimate (4.1).

\subsubsection{Minimizing property of the approximations}

Now, we observe that $u^{(h)}$ is a minimizer of the functional

$$
\mathbf{F}^{(h)}[v]:=\int_{0}^{T} \int_{\Omega}\left[\frac{1}{2 h}\left|v(\cdot, t)-u^{(h)}(\cdot, t-h)\right|^{2}+f\left(g_{v}(\cdot, t)\right)\right] \mathrm{d} \mu \mathrm{d} t
$$

in the class of functions $v \in L^{2}\left(\Omega_{T}\right) \cap\left(\eta^{(h)}+L^{p}\left(0, T ; \mathcal{N}_{0}^{1, p}(\Omega)\right)\right)$. Indeed, for any admissible function $v$ the following computation shows that

$$
\begin{aligned}
\mathbf{F}^{(h)}\left[u^{(h)}\right] & =\sum_{i=1}^{\frac{T}{h}} \int_{(i-1) h}^{i h} \int_{\Omega}\left[\frac{1}{2 h}\left|u_{i}-u_{i-1}\right|^{2}+f\left(g_{u_{i}}\right)\right] \mathrm{d} \mu \mathrm{d} t \\
& =\sum_{i=1}^{\frac{T}{h}} \int_{(i-1) h}^{i h} \mathbf{F}_{i}\left[u_{i}\right] \mathrm{d} t \leq \sum_{i=1}^{\frac{T}{h}} \int_{(i-1) h}^{i h} \mathbf{F}_{i}[v(\cdot, t)] \mathrm{d} t \\
& =\sum_{i=1}^{\frac{T}{h}} \int_{(i-1) h}^{i h} \int_{\Omega}\left[\frac{1}{2 h}\left|v(\cdot, t)-u^{(h)}(\cdot, t-h)\right|^{2}+f\left(g_{v}(\cdot, t)\right)\right] \mathrm{d} \mu \mathrm{d} t \\
& =\mathbf{F}^{(h)}[v]
\end{aligned}
$$

holds true. Here we used the definition of $u^{(h)}$ from (4.6), the minimizing property of $u_{i}$ and the very definition of the functional $\mathbf{F}^{(h)}$. Following the strategy of [7], this leads to the estimate

$$
\begin{aligned}
\int_{0}^{T} & \int_{\Omega} f\left(g_{u^{(h)}}\right) \mathrm{d} \mu \mathrm{d} t \\
\leq & \int_{0}^{T} \int_{\Omega} f\left(g_{v}\right) \mathrm{d} \mu \mathrm{d} t \\
& +\frac{1}{h} \int_{0}^{T} \int_{\Omega}\left(v(\cdot, t)-u^{(h)}(\cdot, t)\right)\left(v(\cdot, t)-u^{(h)}(\cdot, t-h)\right) \mathrm{d} \mu \mathrm{d} t \\
& -\frac{1}{2 h} \int_{T-h}^{T} \int_{\Omega}\left|v-u^{(h)}\right|^{2} \mathrm{~d} \mu \mathrm{d} t \\
& +\frac{1}{2 h} \int_{-h}^{0} \int_{\Omega}\left|v-\eta_{0}\right|^{2} \mathrm{~d} \mu \mathrm{d} t .
\end{aligned}
$$

\subsubsection{The variational inequality for the limit map}

Here we pass to the limit $h \searrow 0$ in (4.11) as was done in [7]. In order to do that we need to replace the boundary condition $v=\eta^{(h)}$ on the lateral boundary by the $h$-independent condition that $v=\eta$. Therefore, we consider a comparison map $v \in \eta+L^{p}\left(0, T ; \mathcal{N}_{0}^{1, p}(\Omega)\right)$ with 
$\partial_{t} v \in L^{2}\left(\Omega_{T}\right), \quad v(0) \in L^{2}(\Omega)$. Again we extend $v$ to negative times by setting $v(\cdot, t):=v(\cdot, 0) \in L^{2}(\Omega)$ for $t<0$. In (4.11) we use $v_{h}:=v+\eta^{(h)}-\eta$ as comparison map. Note that $v_{h} \in L^{2}\left(\Omega_{T}\right) \cap\left(\eta^{(h)}+L^{p}\left(0, T ; \mathcal{N}_{0}^{1, p}(\Omega)\right)\right)$. Since $g_{\eta^{(h)}-\eta} \rightarrow 0$ strongly in $L^{p}(\Omega)$ as $h \searrow 0$, which can be deduced by using the Jensen-type inequality (4.2), we infer by means of the Lipschitz condition (2.9) on $f$ that

$$
\begin{aligned}
& \left|\int_{0}^{T} \int_{\Omega}\left[f\left(g_{v_{h}}\right)-f\left(g_{v}\right)\right] \mathrm{d} \mu \mathrm{d} t\right| \\
& \quad \leq c(p, L) \int_{0}^{T} \int_{\Omega}\left[1+g_{v+\eta^{(h)}-\eta}^{p-1}+g_{v}^{p-1}\right]\left|g_{v+\eta^{(h)}-\eta}-g_{v}\right| \mathrm{d} \mu \mathrm{d} t \\
& \quad \leq c(p, L) \int_{0}^{T} \int_{\Omega}\left[1+g_{v}^{p-1}+g_{\eta^{p-1}-\eta}^{p-1}\right] g_{\eta^{(h)}-\eta} \mathrm{d} \mu \mathrm{d} t \\
& \quad \longrightarrow 0
\end{aligned}
$$

holds true in the limit $h \searrow 0$, i.e. we have

$$
\lim _{h \searrow 0} \int_{0}^{T} \int_{\Omega} f\left(g_{v_{h}}\right) \mathrm{d} \mu \mathrm{d} t=\int_{0}^{T} \int_{\Omega} f\left(g_{v}\right) \mathrm{d} \mu \mathrm{d} t .
$$

In order to treat the other terms appearing in (4.11) we follow again the methods in [7] to find that after passing to the limit $h \searrow 0$ there holds

$$
\begin{aligned}
\int_{0}^{T} \int_{\Omega} f\left(g_{u}\right) \mathrm{d} \mu \mathrm{d} t \leq & \int_{0}^{T} \int_{\Omega} f\left(g_{v}\right) \mathrm{d} \mu \mathrm{d} t+\int_{0}^{T} \int_{\Omega} \partial_{t} v(v-u) \mathrm{d} \mu \mathrm{d} t \\
& -\frac{1}{2}\|(v-u)(T)\|_{L^{2}(\Omega)}^{2}+\frac{1}{2}\left\|v(0)-\eta_{0}\right\|_{L^{2}(\Omega)}^{2}
\end{aligned}
$$

for any comparison function $v \in \eta+L^{p}\left(0, T ; \mathcal{N}_{0}^{1, p}(\Omega)\right)$ with $\partial_{t} v \in L^{2}\left(\Omega_{T}\right)$ and $v(0) \in L^{2}(\Omega)$. This means that $u$ is a variational solution to the Cauchy-Dirichlet problem, which concludes the proof of Theorem 4.1.

\subsection{Approximation by regular data}

In this section we are going to follow [7] and establish the existence result for general boundary data

$$
\eta \in L^{p}\left(0, T ; \mathcal{N}^{1, p}(\Omega)\right)
$$

with

$$
\partial_{t} \eta \in L^{2}\left(\Omega_{T}\right) \text { and } \eta_{0}:=\eta(0) \in L^{2}(\Omega) .
$$

The proof is divided in several steps.

\subsubsection{Regularization}

We consider a sequence $\left(h_{i}\right)_{i \in \mathbb{N}}$ with $h_{i}>0(i \in \mathbb{N})$ and $h_{i} \searrow 0$ as $i \rightarrow \infty$ and let $\varepsilon_{i}:=h_{i}^{\frac{1}{2 p}}$. By [32, Prop. 3.3.49] (see also the proof) we can choose $\eta_{0}^{(i)} \in \operatorname{Lip}(\Omega)$ such that 


$$
g_{\eta_{0}^{(i)}} \leq \frac{1}{\varepsilon_{i}},\left\|\eta_{0}^{(i)}-\eta_{0}\right\|_{L^{2}(\Omega)} \leq \varepsilon_{i}
$$

holds true for all $i \in \mathbb{N}$. Then, we define mollifications in time by

$$
\eta_{i}:=[\eta]_{h_{i}}^{\eta_{0}^{(i)}}
$$

for any $i \in \mathbb{N}$, where $[\cdot]_{h_{i}}^{\eta_{0}^{(i)}}$ is defined as in (3.4). By Lemma 3.6 we have $\eta_{i} \in L^{2}\left(\Omega_{T}\right) \cap L^{p}\left(0, T ; \mathcal{N}^{1, p}(\Omega)\right)$ and according to (3.3) there also holds

$$
\partial_{t} \eta_{i}=\frac{1}{h_{i}}\left(\eta-\eta_{i}\right) \in L^{2}\left(\Omega_{T}\right) \text { and } g_{\partial_{t} \eta_{i}}=\frac{1}{h_{i}} g_{\eta-\eta_{i}} \in L^{p}\left(\Omega_{T}\right) .
$$

For the comparison maps for the limit problem we use the same mollification as for the boundary values $\eta$, i.e. for $v \in \eta+L^{p}\left(0, T ; \mathcal{N}_{0}^{1, p}(\Omega)\right)$ with $\partial_{t} v \in L^{2}\left(\Omega_{T}\right)$ and $v(0) \in L^{2}\left(\Omega_{T}\right)$ we define $v_{i}:=[v]_{h_{i}}^{\eta_{0}^{(i)}}$. By definition, the mollifications satisfy $v_{i} \in \eta_{i}+L^{p}\left(0, T ; \mathcal{N}_{0}^{1, p}(\Omega)\right)$. According to Lemma 3.6 we have that

$$
v_{i} \longrightarrow v \text { in } L^{2}\left(\Omega_{T}\right) \text { in the limit } i \rightarrow \infty .
$$

Next, we claim for all $i \in \mathbb{N}$ that

$$
\int_{0}^{T}\left\|\partial_{t} v_{i}\right\|_{L^{2}(\Omega)} \mathrm{d} t \leq \sqrt{T}\left\|\partial_{t} v\right\|_{L^{2}\left(\Omega_{T}\right)}+\left\|v(0)-\eta_{0}^{(i)}\right\|_{L^{2}(\Omega)},
$$

which implies in particular that the sequence $\left(\partial_{t} v_{i}\right)_{i \in \mathbb{N}}$ is bounded in the space $L^{1}\left(0, T ; L^{2}(\Omega)\right)$. For the proof of this claim we define $\tilde{v}_{i}:=v_{i}+e^{-\frac{\bar{t}}{h_{i}}}\left(v(0)-\eta_{0}^{(i)}\right)$ and note that Lemma 3.7 together with Jensen's inequality implies the bound

$$
\int_{0}^{T}\left\|\partial_{t} \tilde{v}_{i}(t)\right\|_{L^{2}(\Omega)} \mathrm{d} t \leq \sqrt{T}\left\|\partial_{t} \tilde{v}_{i}\right\|_{L^{2}\left(\Omega_{T}\right)} \leq \sqrt{T}\left\|\partial_{t} v\right\|_{L^{2}\left(\Omega_{T}\right)} .
$$

Moreover, we have that

$$
\begin{aligned}
\int_{0}^{T}\left\|\partial_{t}\left(e^{-\frac{t}{h_{i}}}\left(v(0)-\eta_{0}^{(i)}\right)\right)\right\|_{L^{2}(\Omega)} \mathrm{d} t & =\left\|v(0)-\eta_{0}^{(i)}\right\|_{L^{2}(\Omega)} \int_{0}^{T} \frac{1}{h_{i}} e^{-\frac{t}{h_{i}}} \mathrm{~d} t \\
& \leq\left\|v(0)-\eta_{0}^{(i)}\right\|_{L^{2}(\Omega)}
\end{aligned}
$$

holds true for all $i \in \mathbb{N}$. Joining the two preceding estimates, we find that the assertion (4.15) holds true. Our next goal is the proof of the convergence

$$
g_{v_{i}-v} \longrightarrow 0 \text { in } L^{p}\left(\Omega_{T}\right) \text { as } i \rightarrow \infty .
$$

To this end, we consider $[v]_{h_{i}}^{0}=v_{i}-e^{-\frac{t}{h_{i}}} \eta_{0}^{(i)}$. Lemma 3.6 with $r=p$ and $B=\mathcal{N}^{1, p}(\Omega)$ implies

$$
g_{[v]_{h_{i}}^{0}-v} \longrightarrow 0 \text { in } L^{p}\left(\Omega_{T}\right) \text { asi } \rightarrow \infty
$$

Furthermore, we have 


$$
\begin{aligned}
\int_{0}^{T} \int_{\Omega} g_{e^{-t / h_{i}} \eta_{0}^{(i)}}^{p} \mathrm{~d} \mu \mathrm{d} t & =\int_{0}^{T} e^{-\frac{t p}{h_{i}}} \mathrm{~d} t \int_{\Omega} g_{\eta_{0}^{(i)}}^{p} \mathrm{~d} \mu \leq \frac{h_{i}}{p} \int_{\Omega} g_{\eta_{0}^{(i)}}^{p} \mathrm{~d} \mu \\
& \leq \frac{h_{i}}{p} \varepsilon_{i}^{-p} \mu(\Omega)=\frac{\sqrt{h_{i}}}{p} \mu(\Omega) \longrightarrow 0
\end{aligned}
$$

as $i \rightarrow \infty$. Combining (4.18) and (4.19), the assertion (4.17) is verified. The properties (4.16) and (4.17) are in particular valid for the choice $v=\eta$, since the boundary values $\eta$ are admissible as comparison maps.

\subsubsection{Solutions of the regularized problem}

As a consequence of (4.14), the boundary data $\eta_{i}$ and the initial datum $\eta_{0}$ satisfy all assumptions from the preceding steps, i.e. from Sect. 4.1, Theorem 4.1. Hence, we obtain solutions

$$
u_{i} \in L^{\infty}\left(0, T ; L^{2}(\Omega)\right) \cap\left(\eta_{i}+L^{p}\left(0, T ; \mathcal{N}_{0}^{1, p}(\Omega)\right)\right.
$$

of the variational inequalities

$$
\begin{aligned}
\int_{0}^{T} \int_{\Omega} f\left(g_{u_{i}}\right) \mathrm{d} \mu \mathrm{d} t \leq & \int_{0}^{\tau} \int_{\Omega} \partial_{t} v\left(v-u_{i}\right) \mathrm{d} \mu \mathrm{d} t+\int_{0}^{\tau} \int_{\Omega} f\left(g_{v}\right) \mathrm{d} \mu \mathrm{d} t \\
& -\frac{1}{2}\left\|\left(v-u_{i}\right)(\tau)\right\|_{L^{2}(\Omega)}^{2}+\frac{1}{2}\left\|v(0)-\eta_{0}^{(i)}\right\|_{L^{2}(\Omega)}^{2}
\end{aligned}
$$

for a.e. $\tau \in[0, T]$ and every comparison map $v \in \eta_{i}+L^{p}\left(0, T ; \mathcal{N}_{0}^{1, p}(\Omega)\right)$ with $\partial_{t} v \in L^{2}\left(\Omega_{T}\right)$ and $v(0) \in L^{2}(\Omega)$. In particular, we can apply Lemma 3.10 to the variational solutions $u_{i}$ and the comparison function $v=\eta_{i}$ in order to deduce the estimate

$$
\begin{aligned}
\sup _{t \in[0, T]} & \left\|u_{i}(t)\right\|_{L^{2}(\Omega)}^{2}+\int_{0}^{T} \int_{\Omega} f\left(g_{u_{i}}\right) \mathrm{d} \mu \mathrm{d} t \\
\leq & 32\left[\int_{0}^{T}\left\|\partial_{t} \eta_{i}(\cdot, t)\right\|_{L^{2}\left(\Omega_{T}\right)} \mathrm{d} t\right]^{2} \\
& +16 \int_{0}^{T} \int_{\Omega} f\left(g_{\eta_{i}}\right) \mathrm{d} \mu \mathrm{d} t+2 \sup _{t \in[0, T]}\left\|\eta_{i}(t)\right\|_{L^{2}(\Omega)}^{2} .
\end{aligned}
$$

The first two terms on the right-hand side stay bounded in the limit $i \rightarrow \infty$ as a consequence of (4.16) and (4.17) applied to $v=\eta$ and the growth assumption (2.8) 1 on $f$. The last term in (4.21) stays bounded according to Lemma 3.6 with $r=\infty$ and $B=L^{2}(\Omega)$. Hence, the estimate (4.21) together with the coercivity $(2.8)_{1}$ of $f$ implies that the sequence $\left(u_{i}\right)_{i \in \mathbb{N}}$ is bounded in the spaces $L^{\infty}\left(0, T ; L^{2}(\Omega)\right)$ and $L^{p}\left(0, T ; \mathcal{N}^{1, p}(\Omega)\right)$.

\subsubsection{Passage to the limit}

By passing to a subsequence, the results from Sect. 4.2.2 let us achieve convergence

$$
\left\{\begin{array}{l}
u_{i} \rightarrow u \text { weakly in } L^{p}\left(0, T ; \mathcal{N}^{1, p}(\Omega)\right), \\
u_{i} \rightarrow u \text { weak* in } L^{\infty}\left(0, T ; L^{2}(\Omega)\right)
\end{array}\right.
$$

as $i \rightarrow \infty$ for a function $u \in L^{\infty}\left(0, T ; L^{2}(\Omega)\right) \cap L^{p}\left(0, T ; \mathcal{N}^{1, p}(\Omega)\right)$. 
It remains to check that $u$ is a variational solution of the Cauchy-Dirichlet problem. To this end, we consider an arbitrary comparison map $v \in \eta+L^{p}\left(0, T ; \mathcal{N}_{0}^{1, p}(\Omega)\right)$ with $\partial_{t} v \in L^{2}\left(\Omega_{T}\right)$ and $v(0) \in L^{2}(\Omega)$. As before, we write $v_{i}=[v]_{h_{i}}^{\eta_{0}}$ for the time mollification from (3.4) with initial values $\eta_{0}$. By definition the mollifications satisfy $v_{i} \in \eta_{i}+L^{p}\left(0, T ; \mathcal{N}_{0}^{1, p}(\Omega)\right)$. Hence, they are admissible in the variational inequality (4.20), from which we infer

$$
\begin{aligned}
& \frac{1}{2}\left\|\left(v_{i}-u_{i}\right)(\tau)\right\|_{L^{2}(\Omega)}^{2}+\int_{0}^{\tau} \int_{\Omega} f\left(g_{u_{i}}\right) \mathrm{d} \mu \mathrm{d} t \\
& \quad \leq \int_{0}^{\tau} \int_{\Omega} \partial_{t} v_{i}\left(v_{i}-u_{i}\right) \mathrm{d} \mu \mathrm{d} t+\int_{0}^{\tau} \int_{\Omega} f\left(g_{v_{i}}\right) \mathrm{d} \mu \mathrm{d} t
\end{aligned}
$$

for a.e. $\tau \in[0, T]$. Here we used the fact that $v_{i}$ and $\eta_{i}$ possess the same initial datum so that the boundary term at $t=0$ vanishes. Our next aim is to compute the limit of the penultimate integral. By following the procedure in [7] and making use of the properties (4.13) we obtain that

$$
\begin{aligned}
& \lim _{i \rightarrow \infty} \int_{0}^{\tau} \int_{\Omega} \partial_{t} v_{i}\left(v_{i}-u_{i}\right) \mathrm{d} \mu \mathrm{d} t \\
& \quad=\int_{0}^{\tau} \int_{\Omega} \partial_{t} v(v-u) \mathrm{d} \mu \mathrm{d} t+\frac{1}{2}\left\|v(0)-\eta_{0}\right\|_{L^{2}(\Omega)}^{2}
\end{aligned}
$$

holds for a.e. $\tau \in(0, T)$. Furthermore, from (4.16) we infer

$$
\begin{aligned}
& \sup _{i \in \mathbb{N}} \sup _{\tau \in(0, T)}\left|\int_{0}^{\tau} \int_{\Omega} \partial_{t} v_{i}\left(v_{i}-u_{i}\right) \mathrm{d} \mu \mathrm{d} t\right| \\
& \quad \leq \sup _{i \in \mathbb{N}}\left\|\partial_{t} v_{i}\right\|_{L^{1}\left(0, T ; L^{2}(\Omega)\right)}\left\|v_{i}-u_{i}\right\|_{L^{\infty}\left(0, T ; L^{2}(\Omega)\right)}<\infty .
\end{aligned}
$$

Before passing to the limit $i \rightarrow \infty$ in (4.23), we integrate both sides of the inequality over $\tau \in\left(t_{0}, t_{0}+\delta\right) \subset(0, T)$ and take means. This provides us with the estimate

$$
\begin{aligned}
& \frac{1}{2} f_{t_{0}}^{t_{0}+\delta}\left\|\left(v_{i}-u_{i}\right)(\tau)\right\|_{L^{2}(\Omega)}^{2} \mathrm{~d} \tau+\int_{0}^{t_{0}} \int_{\Omega} f\left(g_{u_{i}}\right) \mathrm{d} \mu \mathrm{d} t \\
& \quad \leq f_{t_{0}}^{t_{0}+\delta}\left[\int_{0}^{\tau} \int_{\Omega}\left[\partial_{t} v_{i}\left(v_{i}-u_{i}\right)+f\left(g_{v_{i}}\right)\right] \mathrm{d} \mu \mathrm{d} t\right] \mathrm{d} \tau .
\end{aligned}
$$

The left-hand side is lower semicontinuous as a consequence of (4.22) and (4.15) and the convexity of $f$ together with the monotonicity condition $(2.8)_{2}$. The integral of $f\left(g_{v_{i}}\right)$ converges to the corresponding integral of $f\left(g_{v}\right)$ because of (4.17) and the Lipschitz continuity of $f$ according to (2.9). Finally, the limit of the integral involving the time derivative can be determined with the help of (4.24), (4.25) and the dominated convergence theorem. In this way, we derive the inequality

$$
\begin{aligned}
& \frac{1}{2} f_{t_{0}}^{t_{0}+\delta}\|(v-u)(\tau)\|_{L^{2}(\Omega)}^{2} \mathrm{~d} \tau+\int_{0}^{t_{0}} \int_{\Omega} f\left(g_{u}\right) \mathrm{d} \mu \mathrm{d} t \\
& \quad \leq f_{t_{0}}^{t_{0}+\delta}\left[\int_{0}^{\tau} \int_{\Omega}\left[\partial_{t} v(v-u)+f\left(g_{v}\right)\right] \mathrm{d} \mu \mathrm{d} t\right] \mathrm{d} \tau+\frac{1}{2}\left\|v(0)-\eta_{0}\right\|_{L^{2}(\Omega)}^{2}
\end{aligned}
$$


for every $t_{0} \in(0, T)$ and $\delta \in\left(0, T-t_{0}\right]$. Here, we let $\delta \searrow 0$ to find that

$$
\begin{aligned}
& \frac{1}{2}\left\|(v-u)\left(t_{0}\right)\right\|_{L^{2}(\Omega)}^{2}+\int_{0}^{t_{0}} \int_{\Omega} f\left(g_{u}\right) \mathrm{d} \mu \mathrm{d} t \\
& \quad \leq \int_{0}^{t_{0}} \int_{\Omega}\left[\partial_{t} v(v-u)+f\left(g_{v}\right)\right] \mathrm{d} \mu \mathrm{d} t+\frac{1}{2}\left\|v(0)-\eta_{0}\right\|_{L^{2}(\Omega)}^{2}
\end{aligned}
$$

holds true for a.e. $t_{0} \in[0, T]$. This means that $u$ is the desired variational solution to the Cauchy-Dirichlet problem, which proves Theorem 2.3.

Acknowledgements Open Access funding provided by Projekt DEAL.

Open Access This article is licensed under a Creative Commons Attribution 4.0 International License, which permits use, sharing, adaptation, distribution and reproduction in any medium or format, as long as you give appropriate credit to the original author(s) and the source, provide a link to the Creative Commons licence, and indicate if changes were made. The images or other third party material in this article are included in the article's Creative Commons licence, unless indicated otherwise in a credit line to the material. If material is not included in the article's Creative Commons licence and your intended use is not permitted by statutory regulation or exceeds the permitted use, you will need to obtain permission directly from the copyright holder. To view a copy of this licence, visit http://creativecommons.org/licenses/by/4.0/.

\section{References}

1. Ambrosio, L.: Minimizing movements. R. Accad. Naz. Sci. XL Mem. Mat. Appl. (5) 19, 191-246 (1995)

2. Björn, J.: Poincaré inequalities for powers and products of admissible weights. Ann. Acad. Sci. Fenn. Math. 26, 175-188 (2002)

3. Björn, A., Björn, J.: Nonlinear Potential Theory on Metric Spaces, EMS Tracts in Mathematics. European Mathematical Society, Zurich (2011)

4. Björn, A., Björn, J., Shanmugalingam, N.: The Dirichlet problem for $p$-harmonic functions on metric spaces. J. Reine Angew. Math. 556, 173-203 (2003)

5. Björn, J., Shanmugalingam, N.: Poincaré inequalities, uniform domains and extension properties for Newton-Sobolev functions in metric spaces. J. Math. Anal. Appl. 332, 190-208 (2007)

6. Bögelein, V., Duzaar, F., Marcellini, P.: Parabolic systems with $p, q$-growth: a variational approach. Arch. Ration. Mech. Anal. (1) 210, 219-267 (2013)

7. Bögelein, V., Duzaar, F., Scheven, C.: The obstacle problem for parabolic minimizers. J. Evol. Equ. (4) 17, 1273-1310 (2017)

8. Bögelein, V., Lukkari, T., Scheven, C.: The obstacle problem for the porous medium equation. Math. Ann. (1) 363, 455-499 (2015)

9. Bögelein, V., Scheven, C.: Higher integrability in parabolic obstacle problems. Forum Math. (5) 24, 931-972 (2012)

10. Cheeger, J.: Differentialbility of Lipschitz functions on metric measure spaces. Geom. Funct. Anal. 9, 428-517 (1999)

11. Choe, H.J.: Interior behavior of minimizers for certain functionals with non standard growth. Nonlinear Anal. 19, 933-945 (1992)

12. Cianchi, A., Fusco, N.: Gradient regularity for minimizers under general growth conditions. J. Reine Angew. Mat. 507, 15-36 (1999)

13. Collins, M., Herán, A.: Existence of parabolic minimizers on metric measure spaces. Nonlinear Anal. 176, 56-83 (2018)

14. Dacorogna, B.: Direct Methods in the Calculus of Variations, 2nd edn. Springer, New York (2008)

15. De Giorgi, E.: New Problems on Minimizing Movements. Boundary Value Problems for Partial Differential Equations and Applications. RMA Res. Notes Appl. Math., vol. 29, Masson, Paris, pp. 81-98 (1993)

16. Diestel, J., Uhl, J.J.: Vector Measures, Mathematical Surveys, vol. 15. American Mathematical Society, Providence (1977). (With a foreword by B. J. Pettis) 
17. Esposito, L., Leonetti, F., Mingione, G.: Regularity for minimizers of functionals with $p-q$ growth. Nonlinear Equ. Appl. 6, 133-148 (1999)

18. Franchi, P., Hajlaz, P., Koskela, P.: Definitions on Sobolev classes in metric spaces. Ann. l'inst. Fourier (Grenoble) 49, 1903-1924 (1999)

19. Fujishima, Y., Habermann, J.: Global higher integrability for parabolic quasiminimizers in metric spaces. Adv. Cal. Var. (3) 10, 267-301 (2017)

20. Fujishima, Y., Habermann, J.: The stability problem for parabolic quasiminimizers in metric measure spaces. Rendiconti Lincei-Matematica E Applicazioni XXIX(2), 343-376 (2018)

21. Fujishima, Y., Habermann, J., Kinnunen, J., Masson, M.: Stability for parabolic quasiminimizers. Potential Anal. 41, 983-1004 (2014)

22. Fusco, N., Sbordone, C.: Higher integrability of the gradient of minimizers of functionals with nonstandard growth conditions. Commun. Pure Appl. Math. 43, 673-683 (1990)

23. Grigor'yan, A.A.: The heat equation on noncompact Riemannian manifolds. Mat. Sb. 182, 55-87 (1992). (Translation in Math. USSR-Sb. 72, 47 - 77 (1992))

24. Habermann, J.: Higher integrability for vector-valued parabolic quasi-minimizers on metric measure spaces. Ark. Mat. (1) 54, 85-123 (2016)

25. Hajłasz, P.: Sobolev spaces on an arbitrary metric space. Potential Anal. 5, 403-415 (1996)

26. Hajłasz, P.: Sobolev spaces on metric measure spaces. Heat kernels and analysis on manifolds, graphs and metric spaces. Contemp. Math. 338, 173-218 (2003)

27. Hajłasz, P., Koskela, P.: Sobolev meets Poincaré. C. R. Acad. Sci. Paris Ser. I Math. 320, 12111215 (1995)

28. Hajłasz, P., Koskela, P.: Sobolev met Poincaré. Mem. Am. Math. Soc. 145, x + 101 (2000)

29. Heinonen, J.: Lectures on Analysis on Metric Spaces. Springer, New York (2001)

30. Heinonen, J., Koskela, P.: Quasiconformal maps in metric spaces with controlled geometry. Acta Math. 181, 1-61 (1998)

31. Heinonen, J., Koskela, P.: A note on Lipschitz functions, upper gradients, and the Poincaré inequality. N. Zeal. J. Math. 28, 37-42 (1999)

32. Heinonen, J., Koskela, P., Shanmugalingam, N., Tyson, J.: Sobolev Spaces on Metric Measure Spaces. An Approach Based on Upper Gradients, New Mathematical Monographs, vol. 27. Cambridge University Press, Cambridge (2015)

33. Keith, S., Zhong, X.: The Poincaré inequality is an open ended condition. Ann. Math. (2) 167, 575-599 (2008)

34. Kilpeläinen, T., Kinnunen, J., Martio, O.: Sobolev spaces with zero boundary values on metric spaces. Potential Anal. 12, 233-247 (2000)

35. Kinnunen, J., Marola, N., Miranda Jr., M., Paronetto, F.: Harnack's inequality for parabolic De Giorgi classes in metric spaces. Adv. Differ. Equ. 17, 801-832 (2012)

36. Kinnunen, J., Martio, O.: The Sobolev capacity on metric spaces. Ann. Acad. Sci. Fenn. Math. 21, 367-382 (1996)

37. Kinnunen, J., Shanmugalingam, N.: Regularity of quasi-minimizers on metric spaces. Manuscr. Math. 105, 401-423 (2001)

38. Koskela, P., Shanmugalingam, N., Tuominen, H.: Removable sets for the Poincaré inequality on metric spaces. Indiana Univ. Math. J. 49, 333-352 (2000)

39. Kronz, M.: Some function spaces and spaces of homogeneous type. Manuscr. Math. 106, 219-248 (2001)

40. Laakso, T.: Ahlfors Q-regular spaces with arbitrary Q $>1$ admitting weak Poincaré inequality. Geom. Funct. Anal. 10, 111-123 (2000)

41. Landes, R.: On the existence of weak solutions for quasilinear parabolic initial-boundary value problems. Proc. R. Soc. Edinb. Sect. A (3 - 4) 89, 217-237 (1981)

42. Lichnewsky, A., Temam, R.: Pseudosolutions of the time-dependent minimal surface problem. J. Differ. Equ. (3) 30, 340-364 (1978)

43. Maasalo, O.E., Zatorska-Goldstein, A.: Stability of quasiminimizers of the $p$-Dirichlet integral with varying $p$ on metric spaces. J. Lond. Math. Soc. (2) 77(3), 771-788 (2008)

44. Marcellini, P.: Everywhere regularity for a class of elliptic systems without growth conditions. Ann. Scuola Norm. Sup. Pisa Cl. Sci. 23, 1-25 (1996)

45. Marcellini, P., Papi, G.: Nonlinear elliptic systems with general growth. J. Differ. Equ. 221, 412443 (2006)

46. Marola, N., Masson, M.: On the Harnack inequality for parabolic minimizers in metric measure spaces. Tohoku Math. J. (2) 65, 569-589 (2013)

47. Masson, M., Miranda Jr., M., Paronetto, F., Parviainen, M.: Local higher integrability for parabolic quasiminimizers in metric spaces. Ric. Mat. (2) 62, 279-305 (2013) 
48. Masson, M., Siljander, J.: Hölder regularity for parabolic De Giorgi classes in metric measure spaces. Manuscr. Math. (1) 142, 187-214 (2013)

49. Perlman, M.D.: Jensen's inequality for a convex vector-valued function on an infinite-dimensional space. J. Multivar. Anal. 4, 52-65 (1974)

50. Růžička, M.: Nichtlineare Funktionalanalysis: Eine Einführung, Springer Lehrbuch Masterclass. Springer, Berlin (2004)

51. Saloff-Coste, L.: A note on Poincaré, Sobolev and Harnack inequalities. Int. Math. Res. Not. IMRN 2, 27-38 (1992)

52. Saloff-Coste, L.: Aspects of Sobolev-Type Inequalites, London Mathematical Society Lecture Note Series 289. Cambridge University Press, Cambridge (2002)

53. Shanmugalingam, N.: Newtonian spaces: an extension of Sobolev spaces to metric measure spaces. Rev. Mat. Iberoam. 16(2), 243-279 (2000)

54. Shanmugalingam, N.: Harmonic functions on metric spaces. Ill. J. Math. 45, 1021-1050 (2001)

55. Uhlenbeck, K.: Regularity for a class of nonlinear elliptic systems. Acta Math. 138, 219-240 (1977)

Publisher's Note Springer Nature remains neutral with regard to jurisdictional claims in published maps and institutional affiliations. 